Article

\title{
Coordinated Secondary Frequency Regulation Strategy of Doubly-Fed Induction Generator and Electric Vehicle
}

\author{
Qian Zhang ${ }^{1, * \mathbb{D}}$, Yan $\mathrm{Li}^{1}$, Chen $\mathrm{Li}^{2}$, Zhuwei Ding ${ }^{1}$ and Wenrui Xie ${ }^{1}$ \\ 1 The State Key Laboratory of Power Transmission Equipment \& System Security and New Technology, \\ Chongqing University, Chongqing 400044, China \\ 2 EHV Power Transmission Company, China Southern Power Grid Co Ltd., Guangzhou 510620, China \\ * Correspondence: zhangqian@cqu.edu.cn; Tel.: +86-185-2318-0411
}

Received: 20 June 2019; Accepted: 11 July 2019; Published: 14 July 2019

\begin{abstract}
Wind turbines can participate in frequency regulation by controlling active power output, but the indeterminacy and volatility of wind power result in low reliability of frequency support. Therefore, as a kind of energy storage system, an electric vehicle is adopted to coordinate with wind turbines to regulate system frequency considering its large-scale development. First, based on the reasonable division of wind speed regions and operation point selection of pitch angle, the de-loading strategy of doubly-fed induction generator for reserve capacity under continuously varying wind speed is proposed. Then, through the combination of rotor speed and pitch angle control, frequency regulation model of a doubly-fed induction generator in whole wind speed range is established. Finally, taking into account the driving demand of electric vehicle owners, through the real-time allocation of system frequency regulation task based on frequency regulation capacity, the coordinated control strategy of doubly-fed induction generator and electric vehicle cluster for secondary frequency regulation is put forward. The simulation results show that the coordinated frequency regulation strategy based on real-time allocation can suppress frequency deviation effectively, and the regulation effect is better than the situations of wind turbine coordinating with the conventional unit or coordinating with electric vehicle cluster based on fixed allocation ratio.
\end{abstract}

Keywords: frequency regulation; doubly-fed induction generator; de-loading operation; rotor speed control; pitch angle control; electric vehicle

\section{Introduction}

With the penetration rate of wind power in micro-grid increasing, the indeterminacy and volatility of wind power generation will directly affect the balance of supply and demand of active power in the micro-grid system, causing frequency fluctuations. Countries around the world have introduced grid codes, which stipulate that wind farms must be able to regulate active power and provide frequency support $[1,2]$.

At present, the most widely used kind of wind turbines (WTs) is a double-fed induction generator (DFIG), which is connected to the grid through power electronic devices, thus is unable to respond to system frequency deviations without supplementary active power control methods, which includes rotor speed control, pitch angle control, and their combination. In Ref. [3], only pitch angle control is applied in the frequency regulation (FR) by DFIG, while the potential of rotor speed control is not exploited. However, the power regulation through rotor speed control is faster than pitch angle control, so its FR performance is better. The combination of two control methods is adopted in the FR strategy presented by Ref. [4], but the de-loading of DFIG is not studied; thus, DFIG can only participate in 
down-regulation. Only DFIG in the de-loading state can participate in both system up-regulation and down-regulation.

Constrained by operation limit of DFIG, the de-loading strategy for different wind speed (WS) region is different, and the combination of rotor speed and pitch angle control is commonly applied in the researches about DFIG de-loading. In the DFIG de-loading strategy of Ref. [5], the dividing of WS regions is proposed based on the operation characteristic of DFIG. However, the dividing method does not designate the starting point of low WS region; thus, the FR reserve capacity cannot reach the expected value due to the lower limit of rotor speed. In addition, the pitch angle control is only applied in high WS region, which will result in the lack of reserve capacity in median WS region due to the upper limit of rotor speed. In both reference. [6] and reference [7], the dividing of WS regions is used for appointing different de-loading mode or FR type to WTs under different WS in the wind power plant, which does not consider the smooth transition between control methods. In other words, most existing researches ignored the continuous change of rotor speed or pitch angle at the junctions of different WS regions and the probability of not reaching expected FR reserve capacity in each WS region. Therefore, it is necessary to establish a DFIG FR model, which is applicable under continuously varying WS in whole WS range.

However, WTs underperform in providing FR service due to the volatility and indeterminacy of wind power. Therefore, it is difficult for WT to provide frequency support independently. The energy storage system is often used to assist renewable energy generation to provide auxiliary services [8]. The coordinated control strategy in Ref. [9] uses battery energy storage system (BESS) as a secondary energy resource to assist wind power plant to provide FR service for long-term operation. In Ref. [10], the proposed cooperation strategy employs wind power to track the FR signal in priority and battery storage to compensate for insufficient and inaccurate power. Nevertheless, the auxiliary energy storage system requires a large amount of additional equipment, which will greatly increase the operation cost of the wind farm. Meanwhile, the method only improves the frequency response on the power supply side but does not exploit the potential of the load side in system FR.

The quantity of electric vehicle (EV) will increase significantly in the future because the development of $\mathrm{EV}$ is attached great importance by countries around the world in consideration of the global environmental pollution and energy shortage crisis. Through proper price guidance, EV cluster can provide reserve capacity for FR. As a kind of existing energy storage resources, electric vehicle (EV) can take the place of additional energy storage equipment in assisting WT to participate in FR without extra cost, as well as improve system frequency response on both load and source side at the same time owing to its dual role. In Ref. [11], a charging and discharging control strategy for EV cluster to participate in secondary FR of a two-area interconnected system containing WTs is put forward. An optimal charging/discharging scheduling strategy of EVs is proposed in [12] to improving the frequency stability of micro-grid with penetration of wind power. However, instead of being one of the FR participators, wind power model is only taken as random disturbance of power generation considering its intermittent characteristics in these mentioned researches, which will increase the pressure of system FR.

Therefore, under the background of large-scale wind power integration, it is necessary to appoint a part of WTs in the grid as auxiliary frequency regulation units, sharing FR pressure with EV so as to stabilize system frequency. In Ref. [13], the coordination of EV cluster and WT in system FR is realized by hierarchical control algorithm, in which EV cluster participate in FR in the lower layer, while WT only participates in generation dispatching plan in the upper layer. The coordinated control strategy of WT's pitch angle and EV based on model predictive control is proposed in Ref. [3] for micro-grid FR, but the control imposed on WTs is used for smoothing its power output, so only EV cluster responds to the frequency deviation signal. Two coordinated FR methods for EV and WT based on centralized and distributed control, respectively, are analyzed in Ref. [14], but in the proposed control structures, EV and WT output power for FR separately without the allocation of demand active power between them, which means that the FR tasks of EV and DFIG are not coupled with each other, which may cause the 
system to demand power that cannot be effectively fulfilled or the waste of power output. Moreover, in the most relevant researches, the influence of FR reserve capacity of DFIG or EV on the power output limit is not considered. Therefore, it is necessary to study the allocation method of system FR task considering the FR capacity so as to achieve the mutually coupled coordination of DFIG and EV in FR.

For the sake of solving the problems mentioned above, the main contributions of the paper are listed as follows:

- Through reasonable dividing of WS regions and operation point selection of pitch angle, a modified de-loading strategy of DFIG for FR reserve capacity is proposed, which realizes the continuous change of rotor speed or pitch angle and guarantees expected FR capacity in every WS zone.

- Combining rotor speed and pitch angle control, the FR model of DFIG for both up-regulation and down-regulation under continuously varying WS in whole WS range is established.

- In the premise of meeting the driving demand of EV owners, based on the real-time allocation strategy of system FR task considering the real-time predicted FR capacity of DFIG and EV cluster, the coordinated control strategy of DFIG and EV in system secondary FR is presented.

The remainder of the paper is organized as follows. Section 2 presents the de-loading strategy and FR model of DFIG based on the combination of rotor speed and pitch angle control. Then, Section 3 elaborates the FR control strategy of EV cluster considering the driving demand of vehicle owners. The coordinated FR strategy of EV and DFIG based on the real-time allocation of system FR task is described in Section 4. The analysis of simulation results is shown in Section 5. Finally, conclusions are drawn in Section 6.

\section{Frequency Regulation Model of DFIG}

\subsection{Power Output Control Principle of DFIG}

By controlling the pitch angle and the converters on both sides of rotor and grid, the mechanical power output of wind turbine can be regulated through various controllers $[15,16]$, as shown in (1):

$$
\left\{\begin{array}{l}
P_{m}=\frac{1}{2} \rho_{\text {air }} \pi R^{2} v_{w}^{3} C_{p} \\
C_{p}=c_{1}\left(c_{2} z-c_{3} \beta-c_{4}\right) e^{-c_{5} z} \\
z=\frac{1}{\lambda+c_{6} \beta}-\frac{c_{7}}{1+\beta^{3}} \\
\lambda=\frac{\omega_{M} R}{v_{w}}
\end{array}\right.
$$

where $P_{m}$ is the active power output of DFIG, which equals to the mechanical power captured by DFIG while the loss of gearbox and transmission system is ignored; $\rho_{a i r}$ is the air density, whose value is $1.225 \mathrm{~kg} / \mathrm{m}^{3}$; $R$ is the blade radius of DFIG; $v_{w}$ is the wind speed; $C_{p}$ is the blade power coefficient of DFIG, whose value is related to the pitch angle $\beta$ and the tip-speed ratio $\lambda ; c_{1}-c_{7}$ are relevant parameters of DFIG characteristics [4], whose value are $0.22,116,0.4,5,12.5,0.08$, and 0.035, respectively; $\omega_{M}$ is the angular velocity of blade, whose value is proportional to the rotor speed $\omega_{r}$, as shown in (2):

$$
n=\frac{\omega_{r}}{\omega_{M}}
$$

where $n$ is the gearbox transmission ratio of DFIG, whose value is constant for a fixed WT model.

From Equations (1) and (2), it can be derived that $P_{m}$ changes with wind speed $v_{w}$, rotor speed $\omega_{r}$, and pitch angle $\beta$, which can be expressed as $P_{m}\left(v_{w}, \omega_{r}, \beta\right)$. Therefore, in the situation of fixed WS, the active power output of DFIG can be regulated through controlling the rotor speed and pitch angle. In this way, DFIG will be able to participate in system FR. As can be derived from (1), DFIG blade power coefficient $C_{p}$ changes with $\beta$ and $\lambda$, and Figure 1 shows the change rule of $C_{p}$. It can be seen that when pitch angle $\beta=0^{\circ}$, there is an optimal tip-speed ratio $\lambda_{\text {opt }}$ corresponding to the maximum blade power coefficient $C_{p \text {,max }}$, which can be reached by regulating the rotor speed to be the optimal 
rotor speed $\omega_{\text {opt }}$. Moreover, the DFIG power output can be reduced by increasing the pitch angle to a certain extent.

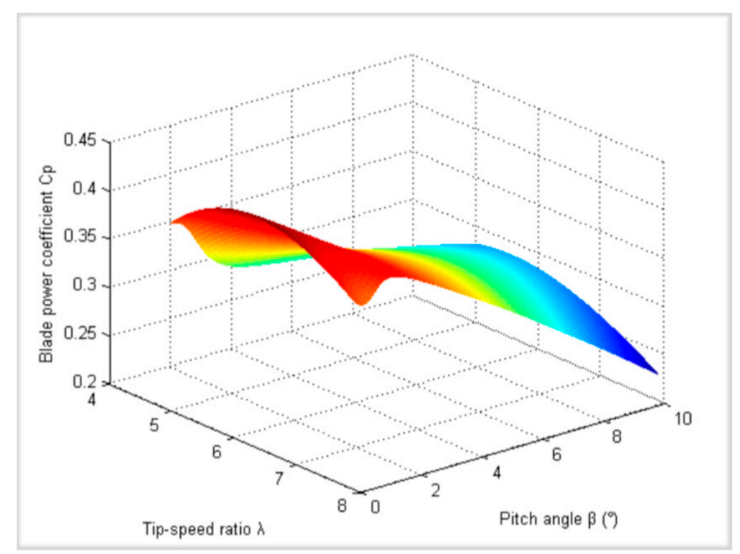

Figure 1. Change rule of DFIG (double-fed induction generator) blade power coefficient.

\subsection{De-Loading Strategy for FR Reserve Capacity}

\subsubsection{Normal Operation of DFIG}

For the normal operation of DFIG, in order to improve the power output efficiency of WT, the maximum power point tracking (MPPT) control strategy is adopted to achieve maximum capture of wind energy [17]. The rotor speed of the DFIG normally operates in the range of 0.7-1.2 p.u., and the safety range can reach 0.6-1.3 p.u. under transient dynamic disturbances [5]. In the MPPT state, the rotor speed of DFIG is adjusted dynamically to be the optimal rotor speed $\omega_{\text {opt }}$, so as to achieve the maximum power output $P_{M P P T}\left(v_{w}\right)$ under different WSs, as shown in Figure 2a. If the maximum power reaches the rated power $P_{e}$, the corresponding optimal speed $\omega_{\text {opt }}$ will go beyond the normal operation range; thus, the pitch angle will be increased to constrain rotor speed within its limit, keeping DFIG's power output at the rated power, as shown in Figure $2 \mathrm{~b}$, where $v_{e}$ is the rated WS corresponding to $P_{e}$.

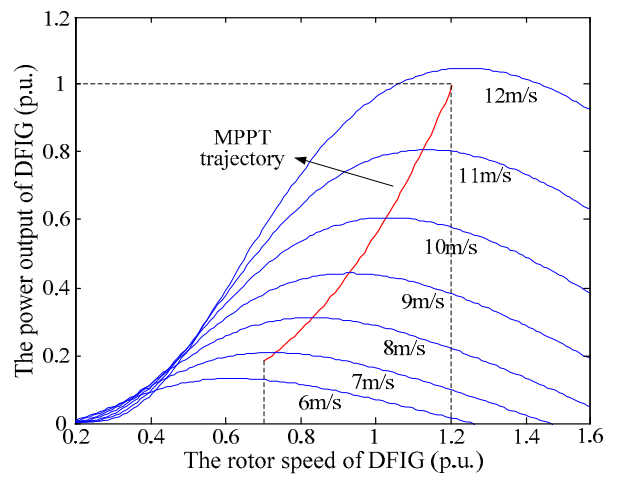

(a)

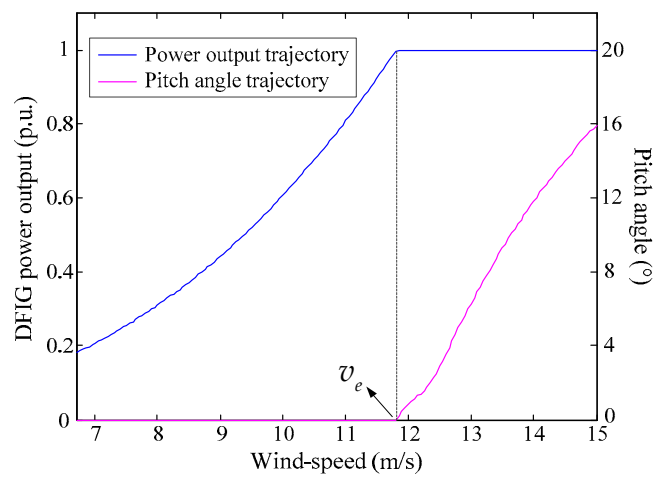

(b)

Figure 2. Normal operation of DFIG (double-fed induction generator). (a) DFIG power output change when $\beta=0^{\circ}$; (b) Normal operation trajectory of power output and pitch angle.

\subsubsection{Division of Wind Speed Region}

For participating in down-regulation, the DFIG can reduce the power output directly through controlling rotor speed or pitch angle. However, DFIG cannot participate in up-regulation when operating in MPPT state since the power output has already reached the maximum value. In order to obtain up-regulation reserve capacity, the DFIG needs to operate in a de-loading state. In order to 
realize the de-loading of DFIG, the rotor speed needs to deviate from the optimal speed. The suboptimal operation state of rotor corresponds to two rotor speeds under the same WS, i.e., the low-speed and over-speed suboptimal rotor speed. In the actual situation, the over-speed suboptimal trajectory is usually adopted in order to maintain DFIG's stable operation.

However, in consideration of the operation limit of rotor speed, the de-loading level may not be satisfied if the power output regulation is only based on rotor speed control. In this situation, the pitch angle control will be activated to keep DFIG power output at a designated de-loading level. In order to realize the smooth transition between rotor speed control, pitch angle control, and their combination in the de-loading strategy, reasonable division of the WS region according to the operation characteristic of DFIG is important.

The whole WS range is divided into three regions considering the operation characteristic of DFIG, including the start-up region, the FR region, and the cut-out region. In the start-up region, the power output of DFIG is too low for providing frequency support; in the cut-out region, the DFIG will exit the grid as the WS is too high. Therefore, the DFIG will only participate in FR in the FR region. Further, considering the operation limit of DFIG, the FR region should be divided into three regions, that is, the low, medium, and high WS region. The division principle of WS regions is elaborated below.

Firstly, in order to ensure that the up-regulation capacity can be fully obtained, the WS at the lower boundary of low WS region should meet the requirement that the maximum point of DFIG power output can be reached when the rotor speed is at the lower limit. Secondly, when suboptimal rotor speed corresponding to designated de-loading level has reached the upper limit, the WS will be set as the boundary of low and median WS region, and it is where the pitch angle control will start to work. Then, the rated WS is set as the boundary of median and high WS region, where the upper limit of rotor speed equals to the optimal speed; thus, only pitch angle control will work on the de-loading in high WS region. Finally, the cut-out speed of DFIG $v_{\text {cut-out }}$ is set as the higher boundary of high WS region. The division principle and corresponding calculated values are shown in Table 1, where $d \%$ is the de-loading rate, which is set to be $10 \%$ in this paper [5] and can be adjusted according to the actual situation.

Table 1. The division of WS (wind speed) regions.

\begin{tabular}{ccc}
\hline WS Region & Division Principle & Boundary Value \\
\hline Start-up \& Low & $P_{m}\left(v_{w}, 0.7,0\right)=P_{M P P T}\left(v_{w}\right)$ & $6.7 \mathrm{~m} / \mathrm{s}$ \\
Low \& Medium & $P_{m}\left(v_{w}, 1.2,0\right)=(1-d \%) P_{M P P T}\left(v_{w}\right)$ & $9.1 \mathrm{~m} / \mathrm{s}$ \\
Medium \& High & $v_{w}=v_{e}$ & $11.8 \mathrm{~m} / \mathrm{s}$ \\
High \& Cut-out & $v_{w}=v_{\text {cut-out }}$ & $25 \mathrm{~m} / \mathrm{s}$ \\
\hline
\end{tabular}

\subsubsection{Operation Point Selection of Pitch Angle}

It is noteworthy that the pitch angle control starts to work at the boundary of low and median WS regions, but the DFIG power output does not increase monotonically with pitch angle here, as shown in Figure 3a, where the current rotor speed keeps at 1.2 p.u. At the boundary WS $(9.1 \mathrm{~m} / \mathrm{s})$, there are three pitch angle values $\left(\beta_{0}, \beta_{1}\right.$ and $\left.\beta_{2}\right)$ that can keep the blade power coefficient $C_{p}$ to be the value corresponding to $(1-d \%) P_{M P P T}\left(v_{w}\right)$, that is, $0.9 C_{p, \max }$. It can be seen that when $\lambda$ decreases with the increase in WS in medium WS region, the change rule of $C_{p}$ with pitch angle is gradually changing from non-monotonic to monotonic. In order to keep the power output at $(1-d \%) P_{M P P T}\left(v_{w}\right)$, the pitch angle will be adjusted to keep $C_{p}$ at $0.9 C_{p \text {,max }}$. However, if $\beta_{0}$ is chosen as the initial operation point of pitch angle in medium WS region, the pitch angle will jump near $9.3 \mathrm{~m} / \mathrm{s}$ due to the change in variation trend of $C_{p}$, as shown in Figure $3 b$. 


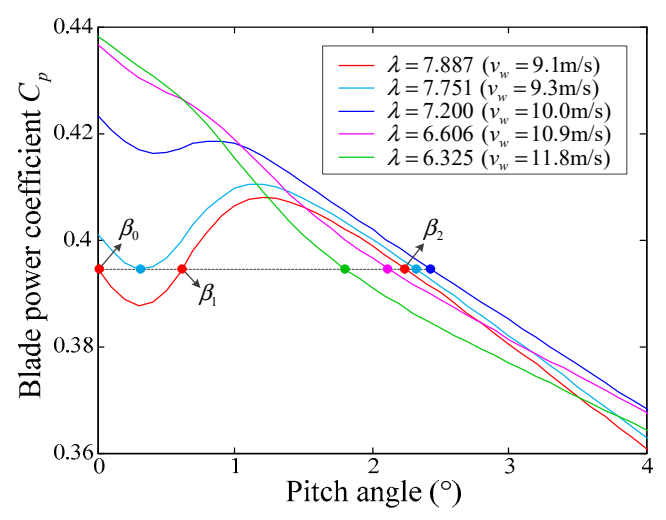

(a)

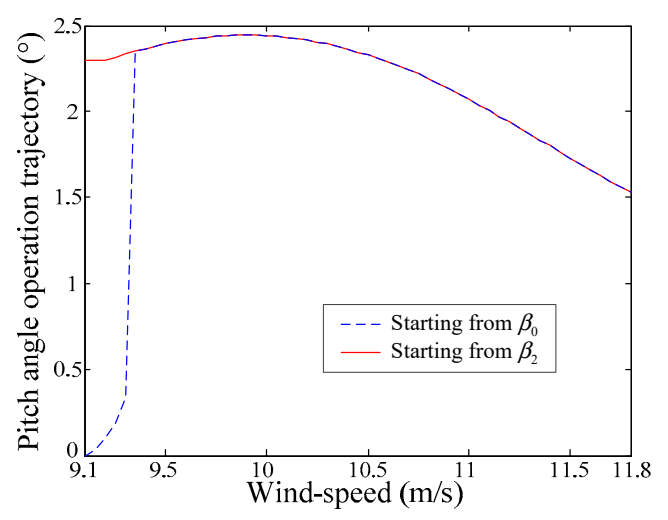

(b)

Figure 3. Analysis of pitch angle change rule. (a) Blade power coefficient change at different WSs (wind speeds); (b) Pitch angle operation trajectory in medium WS region.

On the one hand, the frequent adjustment of pitch angle is not recommended considering the life-span and maintenance cost of WT [7]; on the other hand, when the time interval of FR order is small, the pitch angle may not be able to achieve the target value as a result of change in rate limit. Therefore, for the sake of avoiding the sharp change in pitch angle, $\beta_{2}$ is chosen as the initial operation point of pitch angle control in medium WS region. Moreover, in low WS region, $\lambda$ always keep at 7.887 through the adjustment of rotor speed; thus, the profile of $C_{p}$ is the same as $9.1 \mathrm{~m} / \mathrm{s}$. In order to avoid the jump of pitch angle at the boundary of low and medium WS regions, the pitch angle should keep operating at $\beta_{2}$ in low WS region, which can achieve the same power output as $\beta_{0}$, i.e., $0^{\circ}$.

\subsubsection{De-Loading Strategy of DFIG}

Based on the division of the WS region and operation point selection of pitch angle, the specific de-loading strategy of DFIG in different WS regions are elaborated below.

Low WS region: The DFIG outputs $90 \%$ maximum power by controlling the rotor speed to operate at the over-speed suboptimal trajectory, providing reserve capacity of $10 \%$ maximum power for up-regulation. The pitch angle remains at $\beta_{2}$.

Medium WS region: The rotor speed cannot follow the over-speed suboptimal trajectory due to the upper limit of 1.2 p.u.; thus, the pitch angle is increased to keep $90 \%$ maximum power output, providing reserve capacity of $10 \%$ maximum power for up-regulation. The rotor speed remains at 1.2 p.u.

High WS region: The maximum power point has reached the rated power. The DFIG outputs $90 \%$ rated power by controlling the pitch angle, providing reserve capacity of $10 \%$ rated power for up-regulation. The rotor speed remains at 1.2 p.u.

In the de-loading strategy, the rotor speed and pitch angle operation strategy for providing FR reserve capacity is summed up in (3) and (4), respectively:

$$
\begin{gathered}
\begin{cases}P_{m}\left(v_{w}, \omega_{F R C}, \beta_{2}\right)=(1-d \%) P_{M P P T}\left(v_{w}\right) & 6.7 \mathrm{~m} / \mathrm{s}<v<9.1 \mathrm{~m} / \mathrm{s} \\
\omega_{F R C}=1.2 & 9.1 \mathrm{~m} / \mathrm{s} \leq v<25 \mathrm{~m} / \mathrm{s}\end{cases} \\
\begin{cases}\beta_{F R C}=\beta_{2} & 6.7 \mathrm{~m} / \mathrm{s}<v<9.1 \mathrm{~m} / \mathrm{s} \\
P_{m}\left(v_{w}, 1.2, \beta_{F R C}\right)=(1-d \%) P_{M P P T}\left(v_{w}\right) & 9.1 \mathrm{~m} / \mathrm{s} \leq v \leq 11.8 \mathrm{~m} / \mathrm{s} \\
P_{m}\left(v_{w}, 1.2, \beta_{F R C}\right)=(1-d \%) P_{e} & 11.8 \mathrm{~m} / \mathrm{s}<v<25 \mathrm{~m} / \mathrm{s}\end{cases}
\end{gathered}
$$

where $\omega_{F R C}$ and $\beta_{F R C}$ are the operating rotor speed and a pitch angle of DFIG for providing FR capacity, respectively. In addition, the operation range of the pitch angle is $0-70^{\circ}$ [5]. 
Therefore, through real-time measured wind speed, the FR reserve capacity of DFIG for up-regulation and down-regulation can be predicted in real-time by (5) and (6), respectively:

$$
\begin{gathered}
R_{u p}^{\text {wind }}= \begin{cases}d \% P_{M P P T}\left(v_{w}\right) & 6.7 \mathrm{~m} / \mathrm{s}<v \leq 11.8 \mathrm{~m} / \mathrm{s} \\
d \% P_{e} & 11.8 \mathrm{~m} / \mathrm{s}<v<25 \mathrm{~m} / \mathrm{s}\end{cases} \\
R_{\text {down }}^{\text {wind }}= \begin{cases}\max \left\{P_{\min }, P_{m}\left(v_{w}, 1.2,70^{\circ}\right)\right\}-(1-d \%) P_{M P P T}\left(v_{w}\right) & 6.7 \mathrm{~m} / \mathrm{s}<v \leq 11.8 \mathrm{~m} / \mathrm{s} \\
\max \left\{P_{\min }, P_{m}\left(v_{w}, 1.2,70^{\circ}\right)\right\}-(1-d \%) P_{e} & 11.8 \mathrm{~m} / \mathrm{s}<v<25 \mathrm{~m} / \mathrm{s}\end{cases}
\end{gathered}
$$

where $P_{\min }$ is the lower limit of DFIG's power output for grid integration, whose value equals the maximum power at cut-in speed. If the power output is lower than $P_{\min }$, DFIG's mechanical power cannot maintain the power balance, thus, is unable to participate in FR. Moreover, $R_{u p}^{\text {wind }}$ is set to be positive, and $R_{\text {down }}^{\text {wind }}$ is set to be negative in this paper.

The de-loading operation trajectory of DFIG power output is shown in Figure 4.

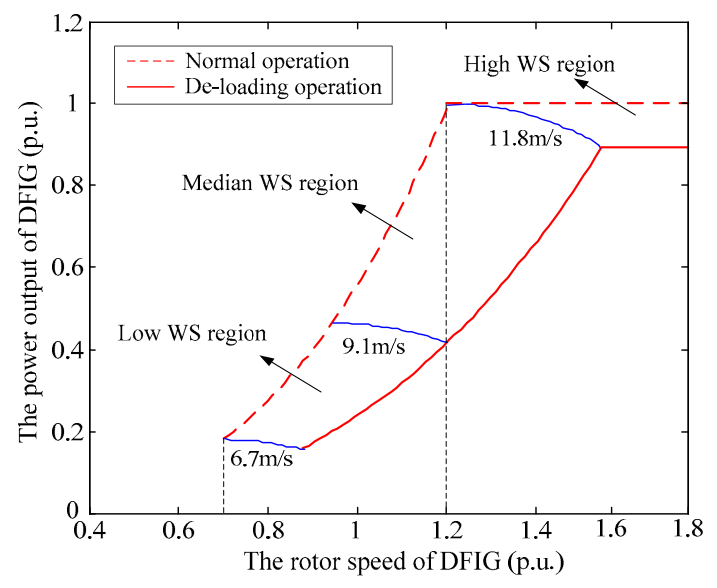

Figure 4. De-loading operation trajectory of DFIG (double-fed induction generator).

\subsection{FR Model of DFIG in Whole WS Range}

Based on the combination of rotor speed and pitch angle control, an FR control model is established for DFIG to participate in system frequency up-regulation and down-regulation. When the system frequency fluctuates, the active power output of DFIG is regulated by controlling rotor speed $\omega_{r}$, pitch angle $\beta$, or both according to the current WS region. The FR control principle block diagram of DFIG is shown in Figure 5. The active power adjustment through rotor speed control is realized by controlling the active component of rotor current; thus, it can respond to the system frequency change in a relatively fast manner. While the pitch angle control is implemented by controlling the mechanical components of the pitch angle controller, which is much slower [7]. Therefore, the rotor speed control is always given priority in the participation of FR.

Based on the operation characteristic and de-loading strategy of DFIG, the FR control methods applied in different WS regions are shown in Table 2. The procedures of DFIG participating in FR in the three WS regions are elaborated below.

\begin{tabular}{|c|c|c|c|}
\hline FR Type WS Region & Low & Medium & High \\
\hline Up-regulation & $\begin{array}{l}\text { Rotor speed control } \\
\text { Pitch angle control }\end{array}$ & $\begin{array}{l}\text { Rotor speed control } \\
\text { Pitch angle control }\end{array}$ & Pitch angle control \\
\hline Down-regulation & $\begin{array}{l}\text { Rotor speed control } \\
\text { Pitch angle control }\end{array}$ & Pitch angle control & Pitch angle control \\
\hline
\end{tabular}

Table 2. FR (frequency regulation) control methods in different WS (wind speed) regions. 


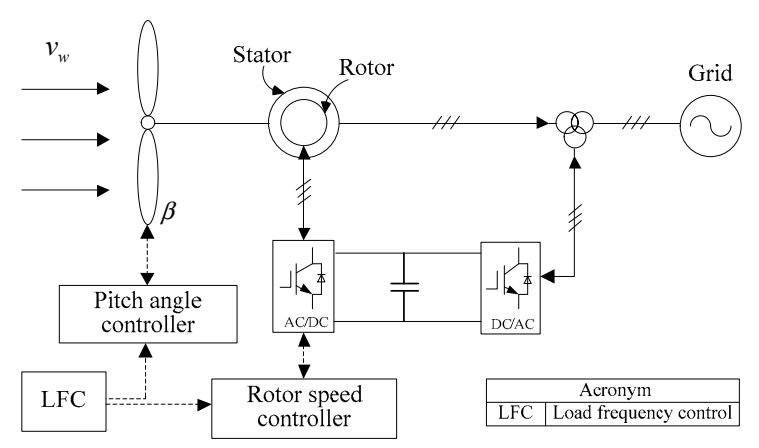

Figure 5. FR (frequency regulation) control principle block diagram of DFIG (double-fed induction generator).

\section{Low WS region:}

Up-regulation: The DFIG reduces $\omega_{r}$ so as to increase power output. When $\omega_{r}$ reaches $\omega_{\text {opt }}$, if the FR task allocated to DFIG by grid control center still cannot be fulfilled, the deceleration should be stopped. Then, $\beta$ will be reduced to continue increasing power output. If the FR task still cannot be fulfilled even when $\beta$ reaches $0^{\circ}, \beta$ stops decreasing and DFIG stays in the MPPT state, providing frequency support in another way.

Down-regulation: The DFIG raises $\omega_{r}$ so as to decrease power output. If the FR task cannot be fulfilled even when $\omega_{r}$ reaches 1.2 p.u., the acceleration should be stopped. Then, $\beta$ will be raised to continue decreasing power output. If the FR task still cannot be fulfilled even when $\beta$ reaches $70^{\circ}$ or the power output reaches $P_{\min }, \beta$ stops increasing, providing frequency support in another way.

\section{Medium WS region:}

Up-regulation: The DFIG reduces $\omega_{r}$ so as to increase power output. If the FR task still cannot be fulfilled even when $\omega_{r}$ reaches $\omega_{\text {opt }}$, the deceleration should be stopped. Then, $\beta$ will be reduced to continue increasing power output. If the FR task still cannot be fulfilled even when $\beta$ reaches $0^{\circ}$, $\beta$ stops decreasing and DFIG stays in the MPPT state, providing frequency support in another way.

Down-regulation: The DFIG raises $\beta$ so as to decrease power output. If the FR task cannot be fulfilled even when $\beta$ reaches $70^{\circ}$ or the power output reaches $P_{\min } \beta$ stops increasing, providing frequency support in another way. $\omega_{r}$ always remains at 1.2 p.u.

\section{High WS region:}

Up-regulation: The DFIG reduces $\beta$ so as to increase power output. If the FR task cannot be fulfilled even when the power output reaches $P_{e}, \beta$ stops decreasing, providing frequency support in another way. $\omega_{r}$ always remains at 1.2 p.u.

Down-regulation: The DFIG raises $\beta$ so as to decrease power output. If the FR task cannot be fulfilled even when $\beta$ reaches $70^{\circ}$ or the power output reaches $P_{\min }, \beta$ stops increasing, providing frequency support in another way. $\omega_{r}$ always remains at 1.2 p.u.

\subsection{Rotor Speed Protection}

In the process of DFIG participating in FR, especially in the low WS region, the excessively large rotor speed drop may cause the DFIG to be unstable or stalled [12]. Therefore, the rotor speed protection is usually introduced to make the DFIG directly exit FR when the rotor speed reaches the lower limit (0.7 p.u.). However, the protection method, with fixed limit value, does not consider the influence of the external WS condition, and DFIG directly exiting FR is also likely to cause a secondary deviation of the system frequency.

Therefore, a rotor speed protection block is added before the DFIG controller in this paper, as shown in Figure 6, where $v_{1}=6.7 \mathrm{~m} / \mathrm{s}$ and $v_{2}=9.1 \mathrm{~m} / \mathrm{s}$. The principle of the protection block is that the FR task of DFIG is gradually reduced with the decrease in WS in low WS region so that the DFIG gradually exits FR with the decrease in rotor speed. The input signal of protection trigger is the system FR task, which ensures the synchronous action of rotor speed protection and DFIG's participation in 
FR. The function of saturation block is set to make the output equal to zero when the input is negative, so the FR task of DFIG will become zero when the WS is below $6.7 \mathrm{~m} / \mathrm{s}$, which means that DFIG is totally exiting FR. In this way, the stability of the wind turbine during the FR process is maintained, and the system frequency is prevented from secondary deviation.

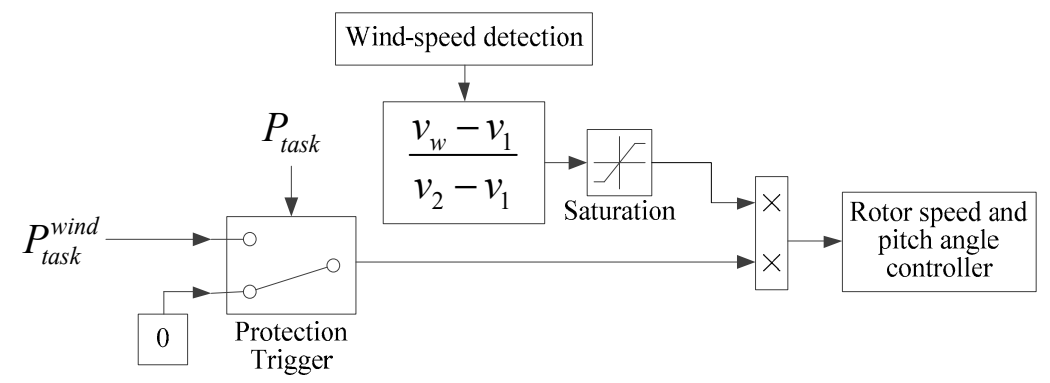

Figure 6. Principle block diagram of rotor speed protection.

In conclusion, based on the de-loading strategy for FR capacity, considering rotor speed control, the secondary FR model for DFIG to participate in both system frequency up-regulation and down-regulation in whole WS range is established through the combination of rotor speed and pitch angle control.

\section{FR Capacity of EV Cluster}

\subsection{Grouping Strategy of EV}

As a kind of mobile energy storage device, EV has the advantage of fast response [18]. EVs can participate in system FR through controlling charging and discharging power [19]. The EV aggregator (EVA) collects the declared information of EVs connected to the grid in real time, including the residence time $T_{i}$ in the grid, the state of charge (SOC), and the credibility $\rho_{i}$. Based on residence time and credibility, EVs connected to the grid are divided into FR service group and energy demand group. In FR service group, according to the initial SOC when EV becomes connected to the grid, EVs are divided into charging group and discharging group. EVA reports the FR capacity of FR service group to control center in real time. According to the system frequency deviation and the FR capacity of DFIG, the grid control center allocates FR task to EVA; then the EVA instructs EVs in the FR service group to charge or discharge to provide frequency support.

Only when the driving demand of vehicle owner is satisfied, EV will participate in FR; otherwise, the EV will be included in the energy demand group, charging to meet the driving demand. The FR service group $S e_{j}$ at time $j$ is shown in (7):

$$
S e_{j}=\left\{i \mid T_{i}>T^{*} \cup \rho_{i} \geq \rho_{\text {down }} \cup j<d_{i}-T_{i}^{D}\right\}
$$

where $T^{*}$ is the threshold of residence time in the grid for FR; $\rho_{\text {down }}$ is the lower threshold of credibility; $d_{i}$ is the planned departure time of the $i$-th EV; $T_{i}^{D}$ is the necessary charging duration for the $i$-th EV to meet driving demand.

After being included in the FR service group, the EV begins to accept the FR instructions from EVA. In order to protect EV battery, frequent conversions between the charging and discharging state, as well as excessive charging/discharging behaviors, should be avoided [20,21]. Therefore, during the FR process, EVs are divided into discharging group $D C$ and charging group $C$ based on the rules shown in Table 3, where $S O C_{i, j}$ is the state of charge of the $i$-th $\mathrm{EV}$ at time $j ; S O C_{\max }$ and $S O C_{\min }$ are the upper and lower limits of SOC, respectively. 
Table 3. Grouping strategy for EVs (electric vehicles) in FR (frequency regulation) service group.

\begin{tabular}{ccl}
\hline Group & Mode & \multicolumn{1}{c}{ Description } \\
\hline Discharging group & 1 & $\begin{array}{l}\text { The EV is included in discharging group. If } S O C_{i, j}>S O C_{\min } \text {, the EV } \\
\text { remains in discharging group and discharges for FR. }\end{array}$ \\
\cline { 2 - 3 } Charging group & 2 & $\begin{array}{l}\text { The EV is included in the charging group. If } S O C_{i, j} \geq S O C_{\max }, \text { the EV } \\
\text { switches into the discharging group and discharges for FR. }\end{array}$ \\
\cline { 2 - 3 } & 3 & $\begin{array}{l}\text { The EV is included in the charging group. If } S O C_{i, j}<S O C_{\max }, \text { the EV } \\
\text { remains in charging group and charges for FR. }\end{array}$ \\
\hline 4 & $\begin{array}{l}\text { The EV is included in discharging group. If } S O C_{i, j} \leq S O C_{\min }, \text { the EV } \\
\text { switches into charging group and charges for } F R . ~\end{array}$ \\
\hline
\end{tabular}

Therefore, the discharging and charging groups at time $j$ are shown in (8) and (9), respectively:

$$
\begin{gathered}
D C_{j}=\left\{i \mid S O C_{i, j} \geq S O C_{\min }, P_{i, j} \geq 0, i \in S e_{j}\right\} \\
C_{j}=\left\{i \mid S O C_{i, j} \leq S O C_{\max }, P_{i, j} \leq 0, i \in S e_{j}\right\}
\end{gathered}
$$

where $P_{i, j}$ is the charging/discharging power for FR of the $i$-th EV at time $j$.

The EV quantity in the discharging and charging groups at time $j$ are shown in (10) and (11), respectively:

$$
\begin{gathered}
N_{j}^{d c}=\left\|D C_{j}\right\| \\
N_{j}^{c}=\left\|C_{j}\right\|
\end{gathered}
$$

\subsection{Real-Time Prediction of EVA's FR Capacity}

As a kind of transportation, EV's traffic characteristics cannot be ignored. It is possible for EV owners to violate dispatching instructions and leaving the grid before the declared departure time, resulting in the inaccurate prediction of EV's FR capacity. When the number of EVs violating the FR dispatching plan reaches a certain level, the FR task allocated to the EVA from the grid cannot be effectively accomplished.

Therefore, in order to solve this problem, the concept of credibility is introduced into the prediction of EV's FR capacity [22]. If an EV obeys the declared plan strictly, leaving the grid according to the declared time, the credibility of this EV is regarded as high; thus, its FR capacity will be fully included in the FR capacity of EVA. However, if an EV is used to suddenly leaving the grid during the declared residence time, the credibility of this EV is regarded as low, and its FR capacity will be included only partially in EVA's FR capacity with the proportion based on the value of credibility. The definition of credibility is shown in (12):

$$
\rho_{i}=\frac{\bar{T}_{i}^{a}}{\bar{T}_{i}^{d}}= \begin{cases}1 & \rho_{\text {up }} \leq \frac{\bar{T}_{i}^{a}}{\bar{T}_{i}^{d}} \\ \bar{T}_{i}^{a} & \rho_{\text {down }} \leq \frac{\bar{T}_{i}^{a}}{\bar{T}_{i}^{d}}<\rho_{\text {up }} \\ 0 & \frac{\bar{T}_{i}^{a}}{\bar{T}_{i}^{d}}<\rho_{\text {down }}\end{cases}
$$

where $\bar{T}_{i}^{a}$ is the historical average actual FR participation time of the EV; $\bar{T}_{i}^{d}$ is historical average declared FR participation time of the EV; $\rho_{u p}$ is the upper threshold of credibility. When the credibility is too high/low, the value is set to be $1 / 0$.

Taking the credibility of EVs into account, the FR capacity of EVA at time $j$ can be predicted in real-time by (13)-(15):

$$
R_{u p, j}^{e v}=\sum_{i=1}^{N_{j}^{d c}}\left(m \cdot \rho_{i}\right)
$$




$$
\begin{gathered}
R_{d o w n, j}^{e v}=\sum_{i=1}^{N_{j}^{c}}\left(-m \cdot \rho_{i}\right) \\
R_{j}^{e v}= \begin{cases}R_{u, j}^{e v} & \Delta f<0 \\
R_{d o w n, j}^{e v} & \Delta f>0 \\
0 & \Delta f=0\end{cases}
\end{gathered}
$$

where $R_{u p, j}^{e v}$ is EVA's real-time predicted capacity for up-regulation; $R_{\text {down, } j}^{e v}$ is EVA's real-time predicted capacity for down-regulation; $m$ is the limit of EV's charging/discharging power; $R_{j}^{e v}$ is the real-time predicted FR capacity of EVA; $\Delta f$ is the system frequency deviation.

\section{The Coordinated FR Strategy of DFIG and EV}

In order to improve the reliability of frequency support provided by DFIGs, considering the frequency stability improvement from load side, EV is used as an auxiliary energy storage system to coordinate with DFIG to participate in system FR.

\subsection{Real-Time Allocation of System FR Task}

In order to realize the coordination of DFIG and EVA in system FR, a real-time allocation strategy of system FR task is proposed. The de-loading operation will influence the economic efficiency of DFIG [4]. Therefore, taking into account the economical operation of the wind farm, the DFIG is set to have priority over EV in up-regulation. Meanwhile, when the EV is connected to the grid, charging is the first demand for EV owner. In order to reduce EV's charging load for driving demand, the EVA is set to have priority over DFIG in down-regulation.

When the system frequency fluctuates, the system FR task $P_{\text {task }}$ will be allocated to DFIG and EVA according to the FR capacity and the type of FR, which includes five modes, as elaborated below. $P_{\text {task }}$ is set to be positive for up-regulation and negative for down-regulation.

Mode 1: If $P_{\text {task }}>0$, the system FR task $P_{\text {task }}$ will be allocated to DFIG first. If the up-regulation capacity of DFIG is more than the system FR task, i.e., $R_{u p}^{\text {wind }} \geq P_{\text {task, }}$, all the FR power output will be provided by DFIG, while EVA will not participate in FR. Thus, the FR tasks of DFIG and EVA are shown in (16):

$$
\left\{\begin{array}{l}
P_{\text {tand }}^{\text {wind }}=P_{\text {task }} \\
P_{\text {task }}^{e v}=0
\end{array}\right.
$$

where $P_{\text {task }}^{\text {wind }}$ and $P_{\text {task }}^{e v}$ are the FR tasks allocated to DFIG and EVA, respectively.

Mode 2: If $P_{\text {task }}>0, P_{\text {task }}$ will be allocated to DFIG first. If $R_{u p}^{\text {wind }}<P_{\text {task}}$, the DFIG cannot accomplish the system FR task alone, so the shortage will be allocated to EVA. Thus, the FR tasks of DFIG and EVA are shown in (17):

$$
\left\{\begin{array}{l}
P_{\text {task }}^{\text {wind }}=R_{\text {up }}^{\text {wind }} \\
P_{\text {task }}^{e v}=P_{\text {task }}-R_{\text {up }}^{\text {wind }}
\end{array}\right.
$$

Mode 3: If $P_{\text {task }}<0, P_{\text {task }}$ will be allocated to EVA first. If the up-regulation capacity of EVA is more than the system FR task, i.e., $R_{\text {down }}^{e v} \leq P_{\text {task }}$, all the FR power output will be provided by EVA, while the DFIG will not participate in FR. Thus, the FR tasks of DFIG and EVA are shown in (18):

$$
\left\{\begin{array}{l}
P_{\text {tank }}^{\text {wind }}=0 \\
P_{\text {task }}^{\text {ev }}=P_{\text {task }}
\end{array}\right.
$$


Mode 4: If $P_{\text {task }}<0, P_{\text {task }}$ will be allocated to EVA first. If $R_{\text {down }}^{e v}>P_{\text {task }}$, the EVA cannot accomplish the system FR task alone, so the shortage will be allocated to DFIG. Thus, the FR tasks of DFIG and EVA are shown in (19):

$$
\left\{\begin{array}{l}
P_{\text {task }}^{e v}=R_{\text {down }}^{e v} \\
P_{\text {task }}^{\text {wind }}=P_{\text {task }}-R_{\text {down }}^{e v}
\end{array}\right.
$$

Mode 5: If $P_{\text {task }}=0$, DFIG and EVA will not participate in FR. Thus, the FR tasks of DFIG and EVA are shown in (20):

$$
\left\{\begin{array}{l}
P_{\text {task }}^{e v}=0 \\
P_{\text {task }}^{\text {wind }}=0
\end{array}\right.
$$

\subsection{The Procedure of Coordinated FR Strategy}

The detailed procedure of DFIG and EVA coordinating to participate in system secondary FR are shown below.

Step 1: According to the WS environment, whether the DFIG can be connected to the grid safely or not will be confirmed. If the security of the DFIG connection can be ensured, the current WS region is determined by the WS, and then the DFIG is controlled to operate in the corresponding de-loading state. At the same time, EVs are connected to the grid according to daily driving rules. Under the dispatch of EVA, EVs in the grid are divided into discharging group, charging group, and energy demand group; then, EV's controllable energy for up-regulation and down-regulation can be calculated in real time.

Step 2: Considering the FR capacity and the type of FR, the system FR task $P_{\text {task }}$ will be allocated to DFIG and EVA according to the proposed real-time allocation strategy.

Step 3: Following the FR task allocated by the control center, both the DFIG and EVA output power to participate in FR.

DFIG: Based on the allocated FR task, the DFIG starts to output power by following the commands from the rotor speed and pitch angle controller. When $P_{\text {task }}^{\text {wind }}$ is accomplished, DFIG stops regulating rotor speed and pitch angle, and the actual power output of DFIG is $P_{a}^{\text {wind }}$. If $P_{\text {task }}^{\text {wind }}$ has not been accomplished when the rotor speed, pitch angle, or output power has reached the operation limit, the DFIG stops regulating its operation state and remains at current power output $P_{a}^{\text {wind }}$.

EVA: Based on the allocated FR task, the EVA instructs the EVs inside the cluster to participate in FR. If the FR capacity of EVA is larger than the FR task of EVA, i.e., $\left|R^{e v}\right| \geq\left|P_{e v}^{\text {task }}\right|$, EVs in the FR service group will be sorted by the credibility $\rho_{i}$ of each EV, and then the EV with a lower credibility will be given higher priority to output power for FR until the FR task is accomplished; if $\left|R^{e v}\right|<\left|P_{e v}^{\text {task }}\right|$, all the EVs in the FR service group will output power for FR at the same time. The actual power output of EVA is $P_{a}^{e v}$.

Step 4: If the system FR task can be accomplished by the actual FR power output of DFIG and EVA, i.e., $\left|P_{a}^{\text {wind }}+P_{a}^{e v}\right| \geq\left|P_{\text {task }}\right|$, the conventional unit will not participate in FR. If the actual FR power output of DFIG and EVA cannot fulfill the system FR task, i.e., $\left|P_{a}^{\text {wind }}+P_{a}^{e v}\right|<\left|P_{\text {task }}\right|$, the shortage will be compensated by the power output of a conventional unit. The FR task allocated to the conventional unit is shown in (21):

$$
P_{\text {task }}^{\text {unit }}=P_{\text {task }}-\left(P_{a}^{\text {wind }}+P_{a}^{e v}\right)
$$

Then, considering the limitation of ramp rate, the conventional unit output power according to the allocated FR task.

In summary, the flow chart of DFIG and EVA coordinating to participate in system FR is shown in Figure 7, where the up-regulation and down-regulation capacity are set to be positive and negative, respectively, for both EVA and DFIG. 


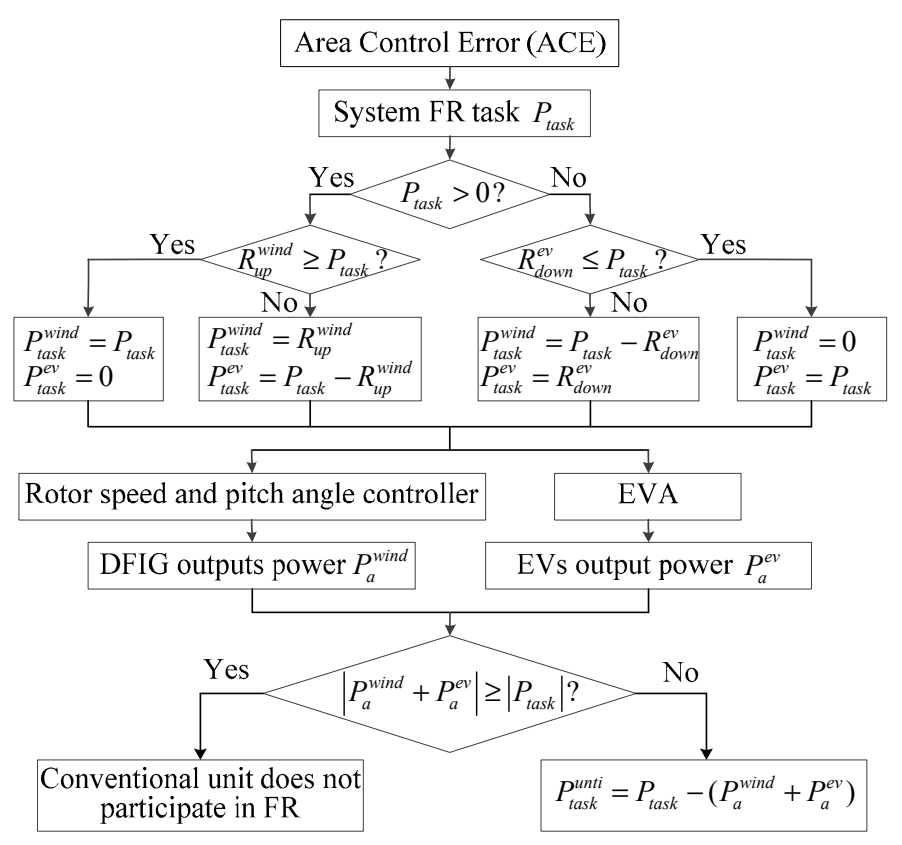

Figure 7. Flow chart of the coordination of DFIG (double-fed induction generator) and EV (electric vehicle) in FR (frequency regulation). EVA: EV aggregator.

\section{Simulation Cases}

\subsection{Simulation Model and Parameters}

The simulation cases in this paper are based on a two-area interconnected system, which contains DFIGs, EV cluster, conventional units, and random loads, as shown in Figure 8. All the simulation results are based on the data of Area 1 . In the system model, $M$ and $D$ are the inertia constant and damping coefficient of the generator, respectively; $1 /\left(s+T_{\text {delay }}\right)$ represents the delay effect of system control and communication, and $T_{\text {delay }}$ is called the system time delay constant. The load frequency control (LFC) blocks in two areas adopt the combination mode of tie-line bias control (TBC), i.e., TBC-TBC mode [23], and the system FR task signal is obtained by proportional-integral (PI) control. The conventional unit block adopts the model of traditional thermal power unit [24].

The parameters of the system model are listed in Table 4 [25]. The parameters of DFIG are listed in Table 5 [26], and the model of DFIGs in two areas are the same. The parameters of EV are listed in Table 6. The driving behaviors and SOC changes of individual EVs are simulated by the Monte Carlo method. By reference to Beijing transport annual report [27], it is assumed that the time of arrival at workplace and time of departure from workplace obey the normal distribution, and the driving distance obeys the lognormal distribution. The system rated frequency $f_{0}$ is $50 \mathrm{~Hz}$. In the simulation results, the base value for the calculation of per-unit value is set to be $5 \mathrm{MW}$.

In the system model, the load fluctuation and WS are simulated through white noise by reference to Ref. [14]. The profiles of load fluctuation and WS in two areas are shown in Figure 9a,b, respectively. In the figure, "p.u." is the abbreviation for per unit, which means the variable is represented in the form of per-unit value. 


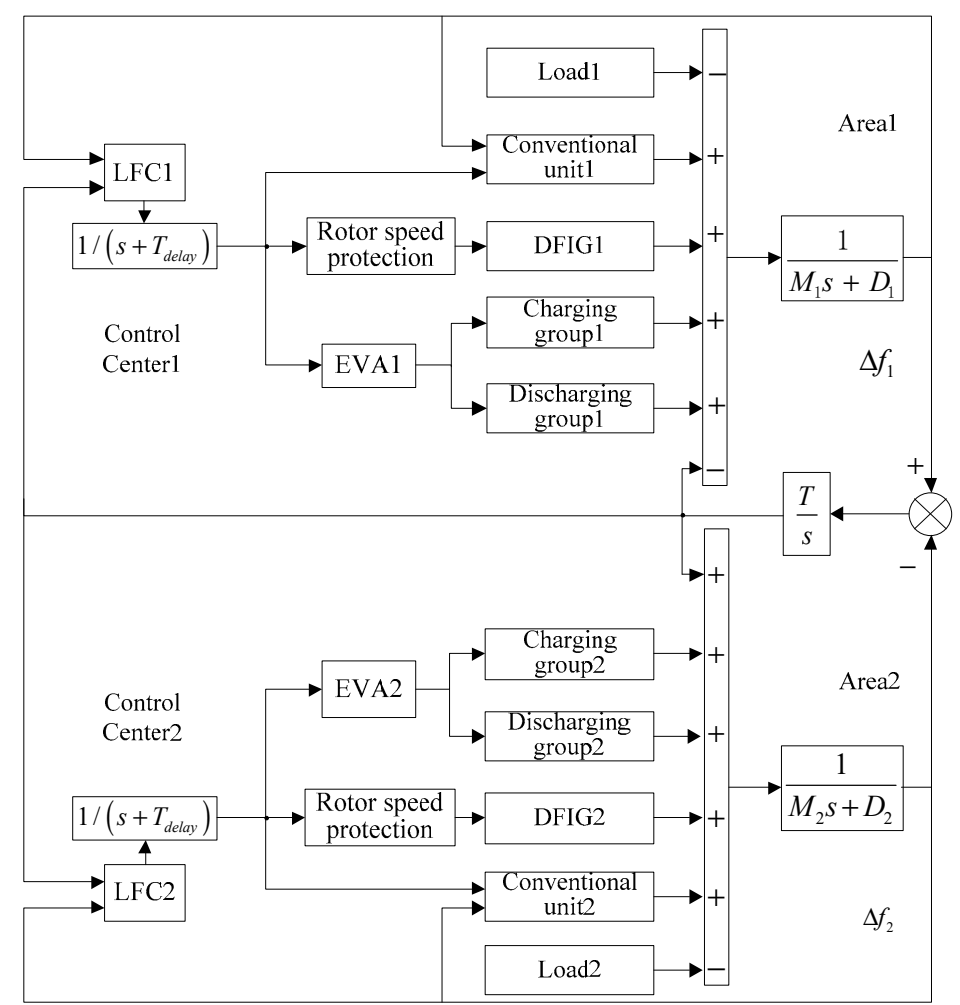

Figure 8. Two-area interconnected system model. LFC: load frequency control; EVA: electric vehicle aggregator; DFIG: double-fed induction generator.

Table 4. Parameters of the two-area interconnected system model.

\begin{tabular}{|c|c|c|}
\hline Parameter Name & Area 1 & Area 2 \\
\hline Generator inertia constant $M(\mathrm{~s})$ & 6.23 & 5.77 \\
\hline Load damping coefficient $D$ (p.u.) & 0.87 & 1.23 \\
\hline Governor speed regulation factor (p.u.) & 0.12 & 0.08 \\
\hline Governor time constant (s) & 0.23 & 0.27 \\
\hline Turbine time constant (s) & 0.56 & 0.45 \\
\hline Frequency deviation factor (p.u.) & 4.89 & 5.12 \\
\hline ACE calculation time constant (s) & 2 & 2 \\
\hline Network-induced delay (s) & \multicolumn{2}{|c|}{1} \\
\hline Tie-line synchronization factor $T$ (p.u.) & \multicolumn{2}{|c|}{2} \\
\hline System time delay constant $T_{\text {delay }}(\mathrm{s})$ & \multicolumn{2}{|c|}{1} \\
\hline
\end{tabular}

ACE: area control error.

Table 5. Parameters of DFIG (double-fed induction generator).

\begin{tabular}{cc}
\hline Parameter Name & Parameter Value \\
\hline Power factor & 1 \\
Polar number & 3 \\
Rated power & $5 \mathrm{MW}$ \\
Rated speed & $1000 \mathrm{rpm}$ \\
Gearbox transmission ratio $n$ & 100 \\
Blade radius $R$ & $60 \mathrm{~m}$ \\
Inertia constant & $1.12 \mathrm{~s}$ \\
The optimal tip-speed ratio & 6.325 \\
Pitch angle change rate limit & $\pm 8^{\circ} / \mathrm{s}$ \\
Cut-in speed & $4 \mathrm{~m} / \mathrm{s}$ \\
Rated wind speed & $11.8 \mathrm{~m} / \mathrm{s}$ \\
Cut-out speed & $25 \mathrm{~m} / \mathrm{s}$ \\
\hline
\end{tabular}


Table 6. Parameters of EV (electric vehicle).

\begin{tabular}{cc}
\hline Name & Parameter \\
\hline Threshold of residence time $T^{*}$ & $1 \mathrm{~h}$ \\
Charging/discharging efficiency & 0.95 \\
Battery capacity & $30 \mathrm{kWh}$ \\
Initial SOC & $S O C_{0} \sim \mathrm{U}(0.2,0.8)$ \\
$S O C_{\max }$ & 0.9 \\
$S O C_{\min }$ & 0.2 \\
Time of arrival at workplace $A R$ & $A R \sim \mathrm{N}(8.5,0.52)$ \\
Time of departure from workplace $D P$ & $D P \sim \mathrm{N}(17.5,0.52)$ \\
Driving distance $D S(\mathrm{~km})$ & $\ln D S \sim \mathrm{N}(17.9,4.9)$ \\
Driving speed [28] & $28.5 \mathrm{~km} / \mathrm{h}$ \\
Energy consumption & $15(\mathrm{kWh} / 100 \mathrm{~km})$ \\
$\rho_{\text {up }}$ & 0.95 \\
$\rho_{\text {down }}$ & 0.15 \\
Vehicle number & 1000 \\
\hline
\end{tabular}

SOC: state of charge.

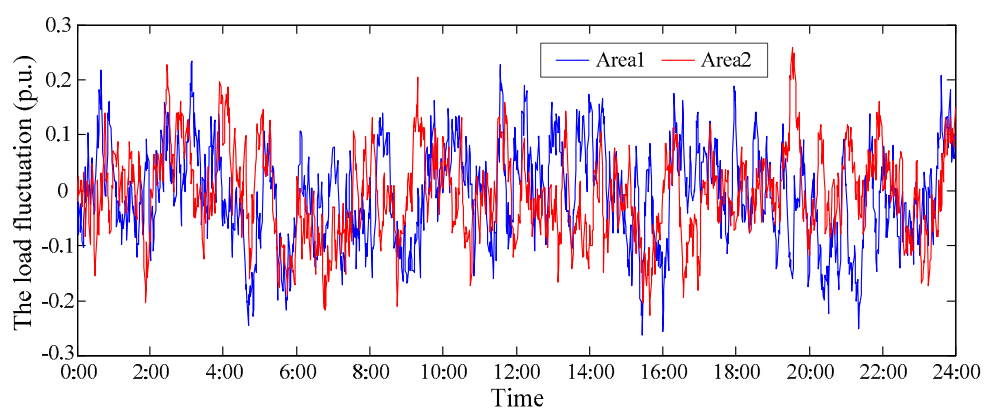

(a)

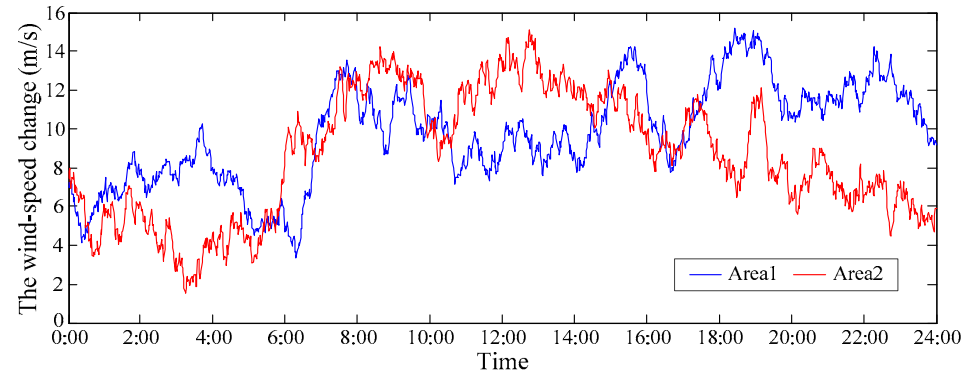

(b)

Figure 9. Profiles of load fluctuation and wind speed in the two-area model. (a) Profiles of load fluctuation; (b) Profiles of wind speed.

\subsection{DFIG Participating in FR}

In order to verify the effectiveness of the proposed FR model for DFIG, two scenarios are set up. In Scenario 1, DFIG participates in FR, and the lack of FR demand power is compensated by the conventional unit. In Scenario 2, DFIG does not participate in FR and operates according to the proposed de-loading strategy. Other conditions of the two scenarios are the same.

The power output of DFIG in two scenarios are shown in Figure 10. Following the proposed de-loading operation strategy for FR capacity, DFIG's power output in Scenario 2 varies with the change in WS. It can be seen from the figure that DFIG's power output in Scenario 1 fluctuates around the de-loading operation curve in Scenario 2, which indicates that DFIG plays an active role in system FR through regulating power output. 


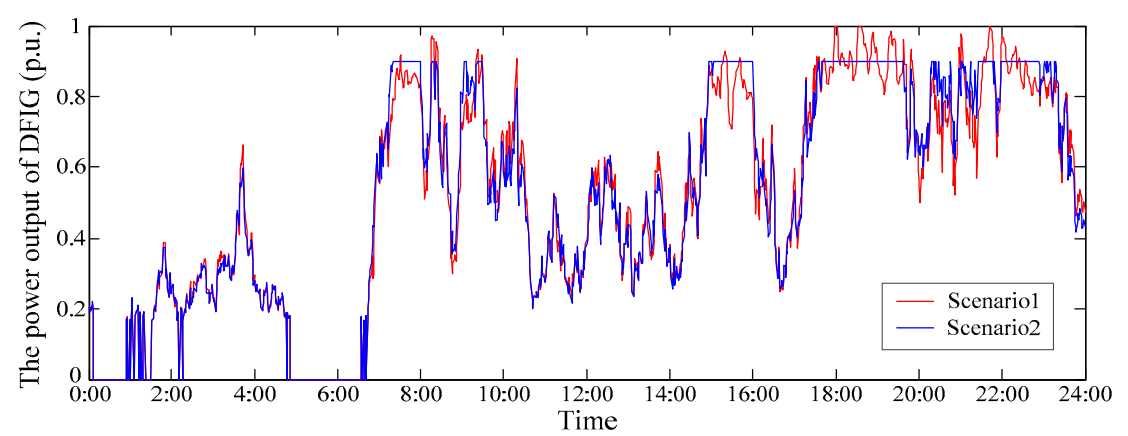

Figure 10. The Power output of the DFIG (double-fed induction generator) during FR (frequency regulation) process.

The FR task of DFIG in Scenario 1 is shown in Figure 11, where the blue curve represents the FR task without rotor speed protection, while the red curve represents the FR task after being processed by rotor speed protection block. It can be seen that in the time period of 2:00-5:00 and 10:30-14:30, the WS is in the low-speed region, as shown in Figure 9b; thus, the rotor speed protection will work and reduce DFIG's FR task with the decrease in WS. In the time period of 0:00-2:00 and 5:00-7:00, the WS is lower than $6.7 \mathrm{~m} / \mathrm{s}$, so DFIG completely exits FR, and its FR task is set to be zero.

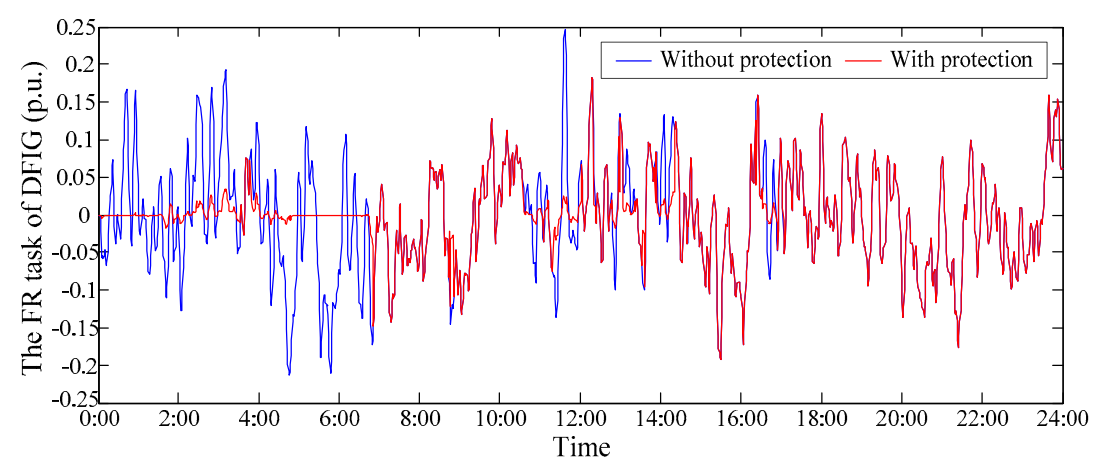

Figure 11. FR (frequency regulation) task of DFIG (double-fed induction aggregator).

The FR capacity of DFIG for up-regulation and down-regulation are shown in Figure 12a,b, respectively. It can be seen that in low and medium WS region, the up-regulation capacity of DFIG is relatively less when the WS is small and rises with the increase in WS. When the WS enters high WS region, the up-regulation capacity remains at 0.1 p.u. At the same time, the down-regulation capacity of DFIG rises as well with the increase in WS, and DFIG's down-regulation capacity is more sufficient than up-regulation capacity due to the operation characteristic of DFIG and the set value of de-loading rate.

The FR output of DFIG is shown in Figure 13. The comparison of FR output and FR task is shown in Figure 13a. It can be seen that the FR task is well accomplished in the down-regulation situation since the FR capacity for down-regulation is sufficient, but the FR task for up-regulation is not completely accomplished. This is because, in the situation of up-regulation, the FR output of DFIG is restrained by the FR capacity, as shown in Figure 13b.

The operation state changes of DFIG are shown in Figure 14, where the rotor speed or pitch angle equaling to zero indicates that the DFIG has exited FR. The rotor speed change in the FR process is shown in Figure 14a. Corresponding to the WS profile, it can be seen that in low WS region, $\omega_{F R C}$ in Scenario 2 changes to keep the power output at $0.9 P_{M P P T}\left(v_{w}\right)$. On this basis, $\omega_{r}$ in Scenario 1 changes to regulate DFIG's power output for FR. In medium and high WS regions, $\omega_{F R C}$ remains at 1.2 p.u., and $\omega_{r}$ only decrease to participate in up-regulation except for transient over-limit. Change in $\beta$ in the FR process is shown in Figure 14b. In low WS region, $\beta_{F R C}$ in Scenario 2 keeps at $\beta_{2}=2.3^{\circ}$; in medium 
and high WS regions, $\beta_{F R C}$ will increase to keep power output at $0.9 P_{M P P T}\left(v_{w}\right)$ and $0.9 P_{e}$, respectively. In Scenario 1, $\beta$ changes to participate in both up-regulation and down-regulation.

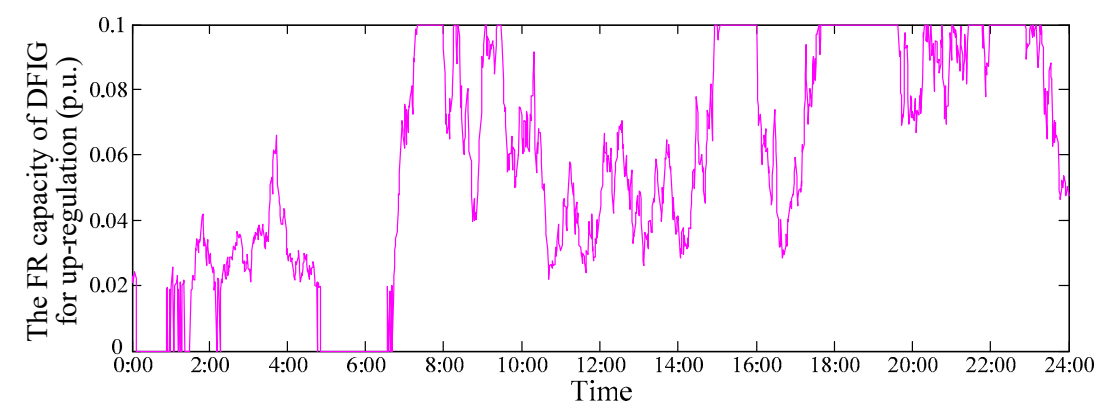

(a)

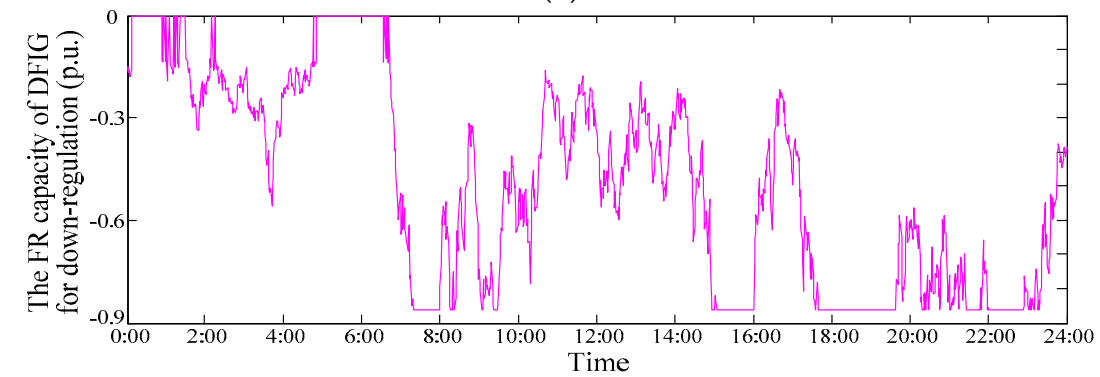

(b)

Figure 12. FR (frequency regulation) capacity of DFIG (double-fed induction generator). (a) DFIG's FR capacity for up-regulation; (b) DFIG's FR capacity for down-regulation.

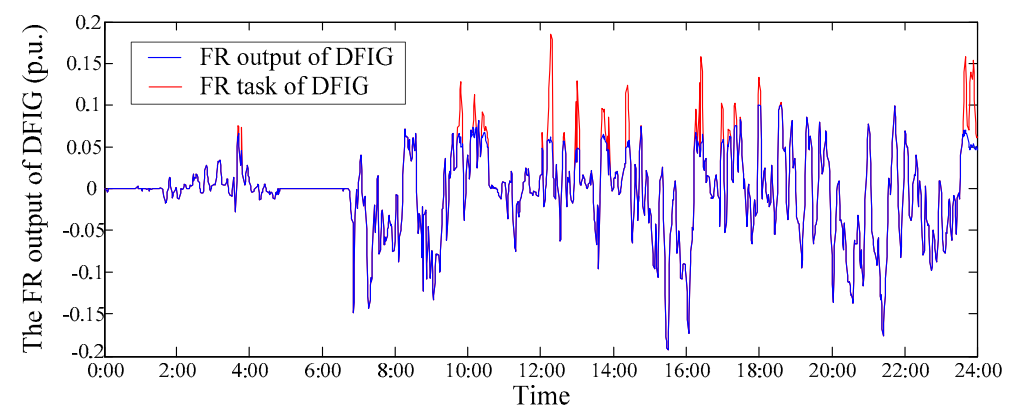

(a)

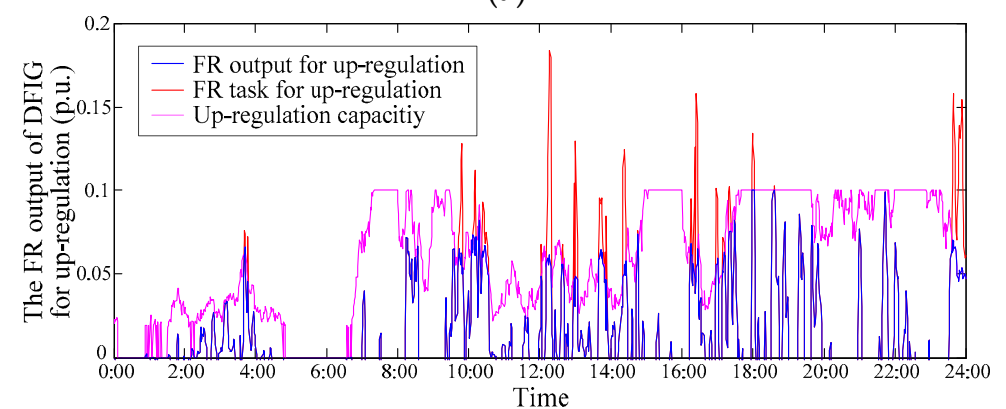

(b)

Figure 13. FR (frequency regulation) output of DFIG (double-fed induction generator). (a) DFIG's output for FR; (b) DFIG's output for up-regulation. 


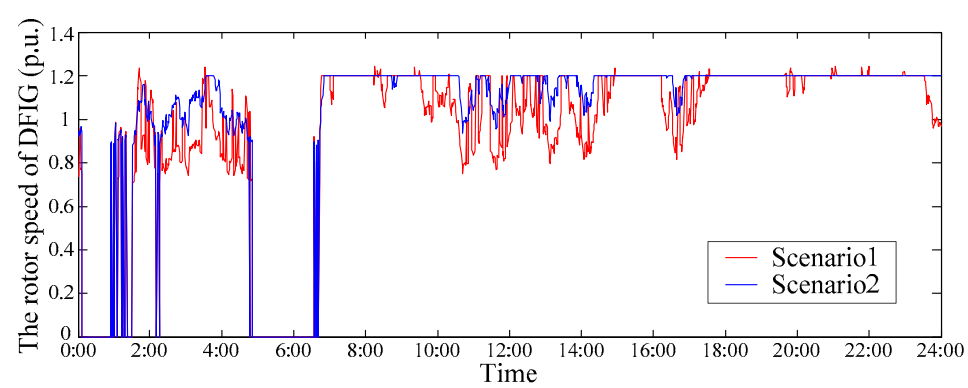

(a)

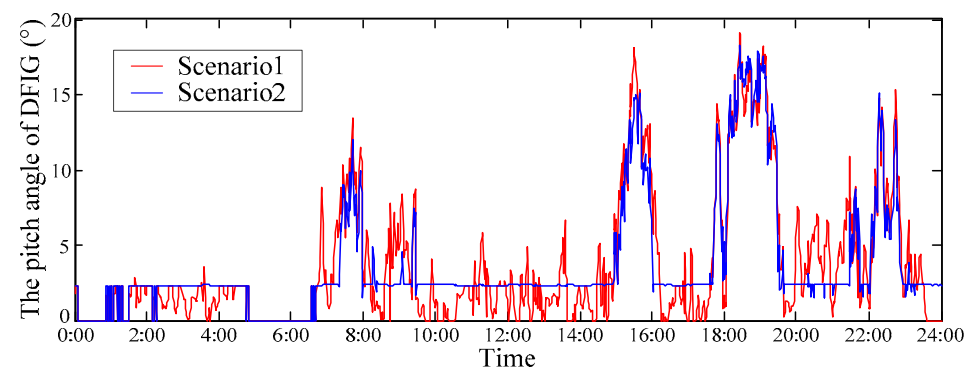

(b)

Figure 14. The Operation state change of DFIG (double-fed induction generator). (a) The rotor speed change of DFIG; (b) The pitch angle change of DFIG.

The comparison of the FR effect in the two scenarios is shown in Figure 15. The root mean square (RMS) value of frequency deviation in Scenario 1 and Scenario 2 is $0.0346 \mathrm{~Hz}$ and $0.0386 \mathrm{~Hz}$, respectively. It can be seen that the FR effect of DFIG participating in FR is obviously better than the situation without frequency support by DFIG, especially in medium and high WS regions. In low WS region, the FR effect is relatively worse as the FR output of DFIG is restrained by both the rotor speed protection and limit of FR capacity. In conclusion, the proposed FR model of DFIG can suppress system frequency deviation effectively under continuously varying WS.

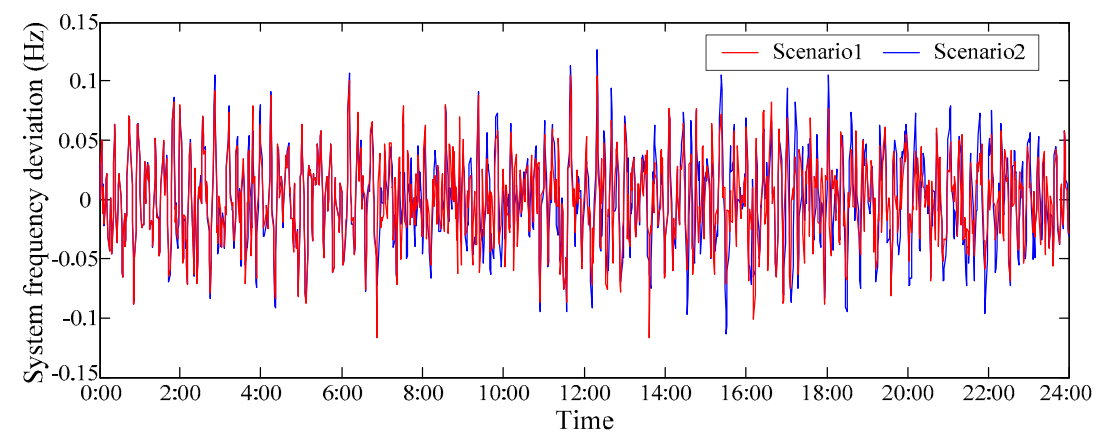

Figure 15. Comparison of FR (frequency regulation) effect in Scenario 1 and Scenario 2.

\subsection{DFIG and EV Coordinating to Participate in FR}

In order to verify the effectiveness of the coordinated FR strategy of DFIG and EV, Scenario 3 is set up. In Scenario 3, DFIG and EV cluster coordinates to participate in system FR, and the lack of FR demand power is compensated by the conventional unit. Other conditions are the same as with Scenario 1 and Scenario 2. The system FR task in Scenario 3 is shown in Figure 16, which will be allocated to EV and DFIG, respectively, according to the proposed real-time allocation strategy. 


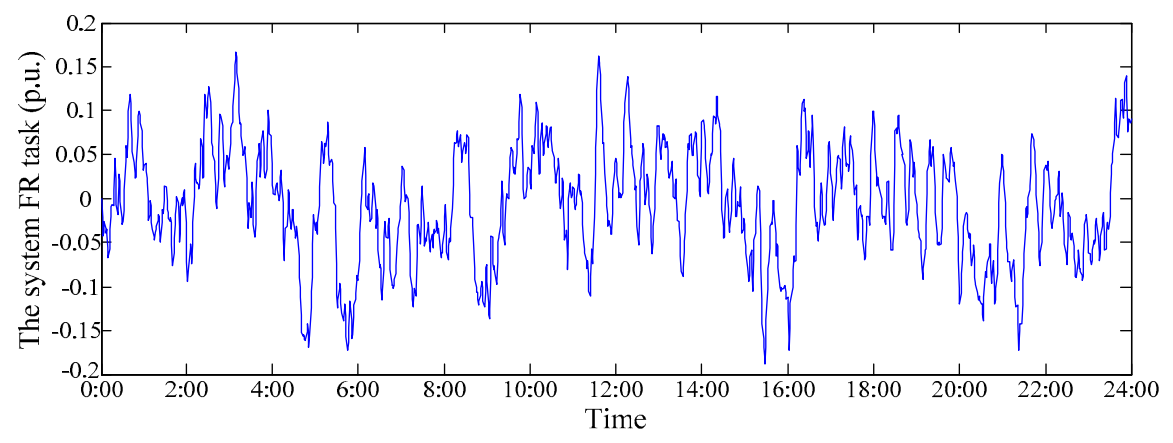

Figure 16. System FR (frequency regulation) task.

The FR capacity of EV cluster for up-regulation and down-regulation is shown in Figure 17a,b, respectively. With EVs accessing and leaving the grid according to the driving rules, the FR capacity of EV cluster will vary with time. The FR output of EV cluster for up-regulation and down-regulation is shown in Figure 18a,b, respectively. It can be seen from the figure that as EVA is given priority in down-regulation in the proposed FR task allocation strategy, EVA's down-regulation task is more than up-regulation task. EV cluster will participate in up-regulation only when the up-regulation capacity of EVA cannot fulfill the FR task. In 9:00-10:00 of Figure 18a as well as 8:30-9:00 and 17:30-19:00 of Figure $18 \mathbf{b}$, the FR output of EV cluster is constrained by the FR capacity.

The FR output of DFIG is shown in Figure 19. It can be seen from the figure that as DFIG is given priority in up-regulation in the proposed FR task allocation strategy, DFIG's up-regulation task is more than down-regulation task. DFIG will participate in down-regulation only when the down-regulation capacity of EVA cannot fulfill the FR task. The FR task allocated to DFIG is almost accomplished, except for some up-regulation moment that the FR output of DFIG is constrained by the FR capacity.

If the joint FR output of EV cluster and DFIG cannot fulfill the system FR task, the conventional unit will output power to compensate for the lack of FR demand power. The FR task allocated to the conventional unit is shown in Figure 20.

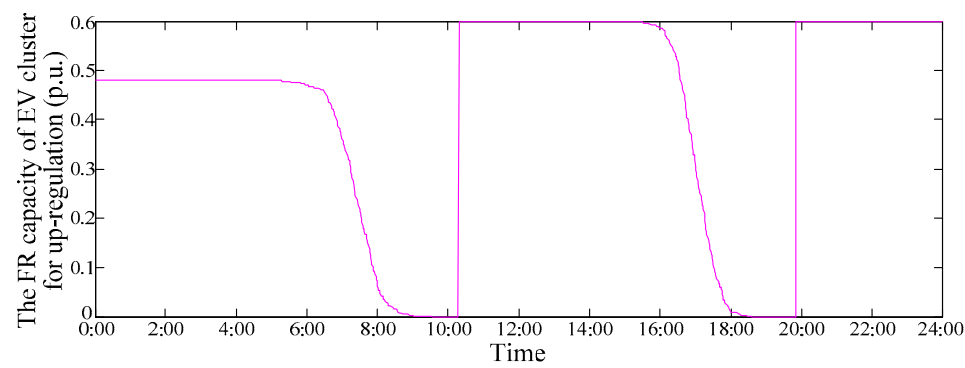

(a)

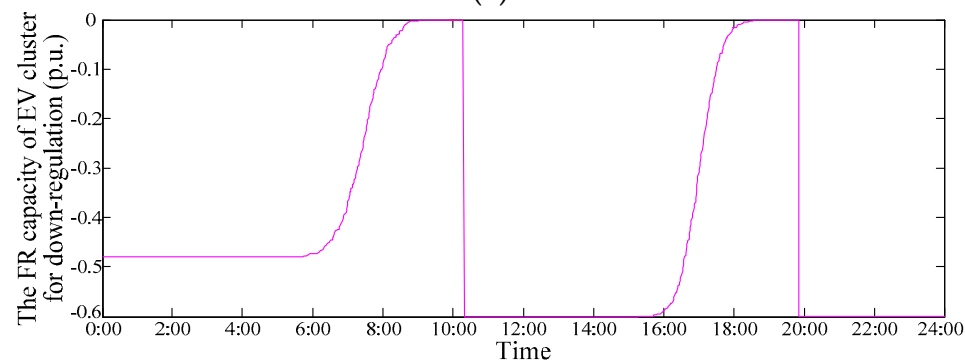

(b)

Figure 17. FR (frequency regulation) capacity of EV (electric vehicle) cluster. (a) EV's FR capacity for up-regulation; (b) EV's FR capacity for down-regulation. 


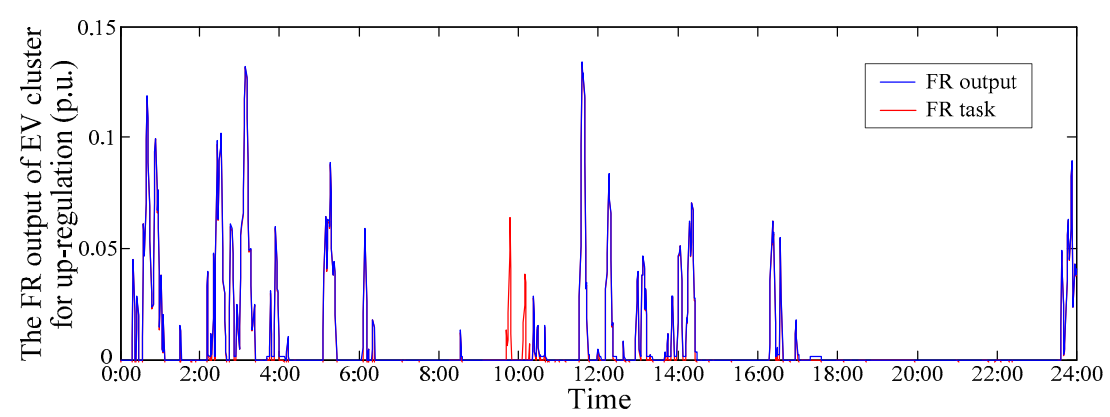

(a)

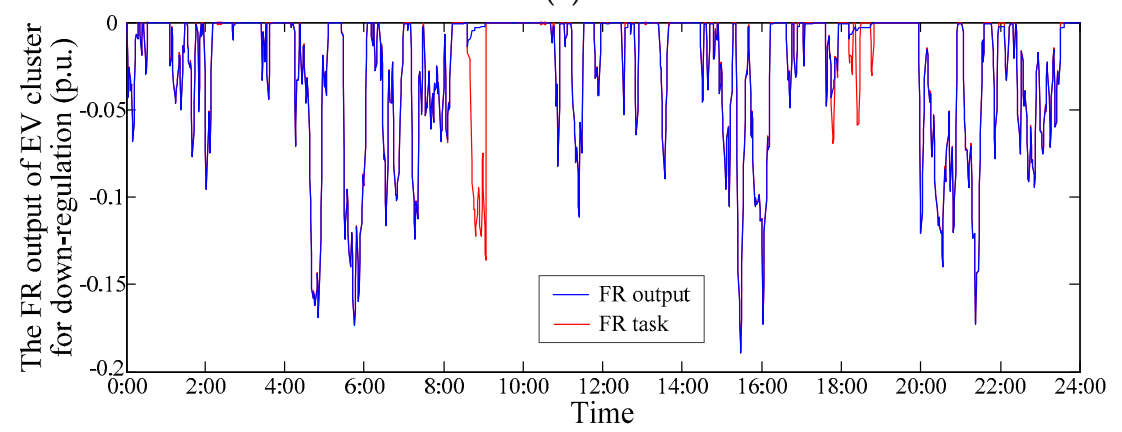

(b)

Figure 18. FR (frequency regulation) output of EV (electric vehicle) cluster. (a) EV's FR output for up-regulation; (b) EV's FR output for down-regulation.

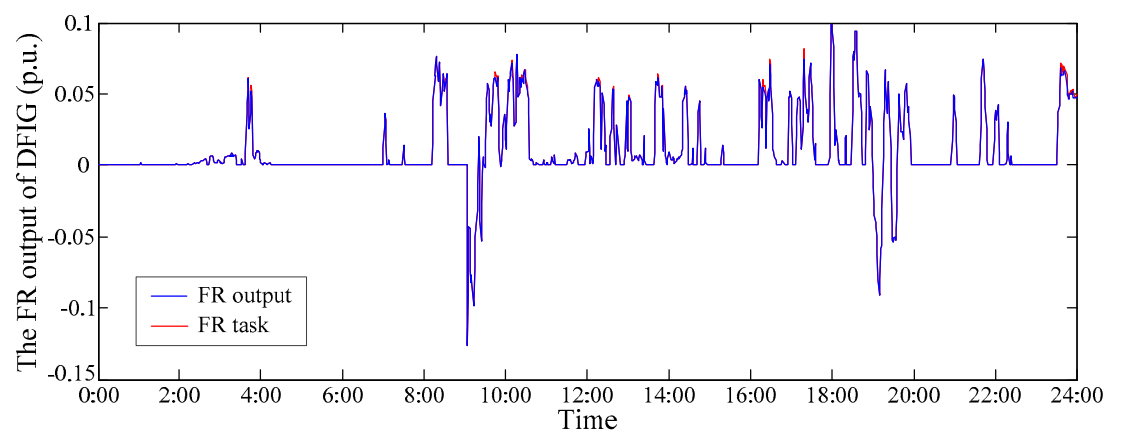

Figure 19. FR (frequency regulation) output of DFIG (double-fed induction generator).

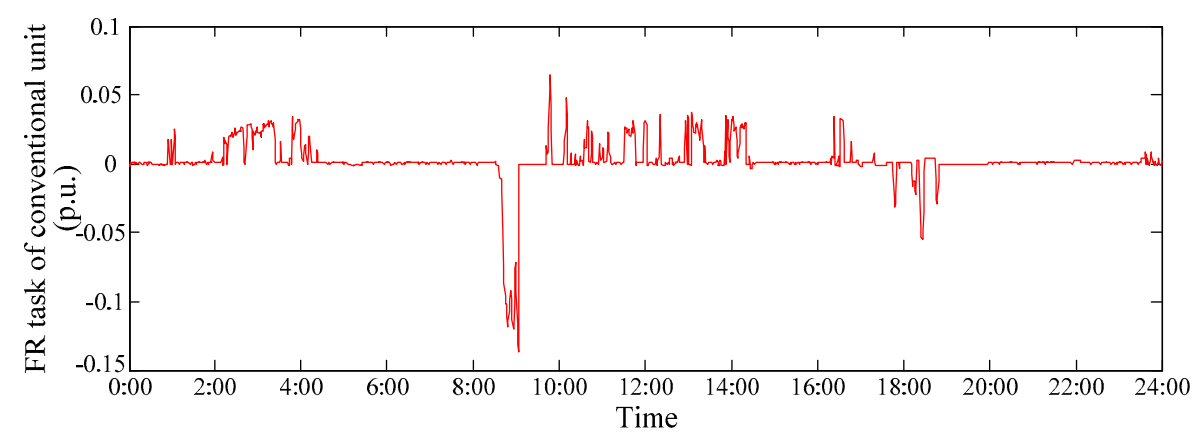

Figure 20. FR (frequency regulation) task of a conventional unit.

In Scenario 1, DFIG coordinates with the conventional unit to participate in FR, while in Scenario 3, DFIG coordinates with EVA to participate in FR. The comparison of the FR effect in Scenario 1 and Scenario 3 is shown in Figure 21. The RMS value of frequency deviation in Scenario 1 and Scenario 3 is $0.0346 \mathrm{~Hz}$ and $0.0267 \mathrm{~Hz}$, respectively. It can be seen that the FR effect of DFIG coordinating with EVA is obviously better than the situation when DFIG is coordinating with the conventional unit. This is because EVs can respond to the FR signal more quickly than the conventional unit, so the system FR 
task can be better accomplished. Therefore, through the proposed coordinated FR strategy, EV cluster is able to assist DFIG to participate in FR effectively.

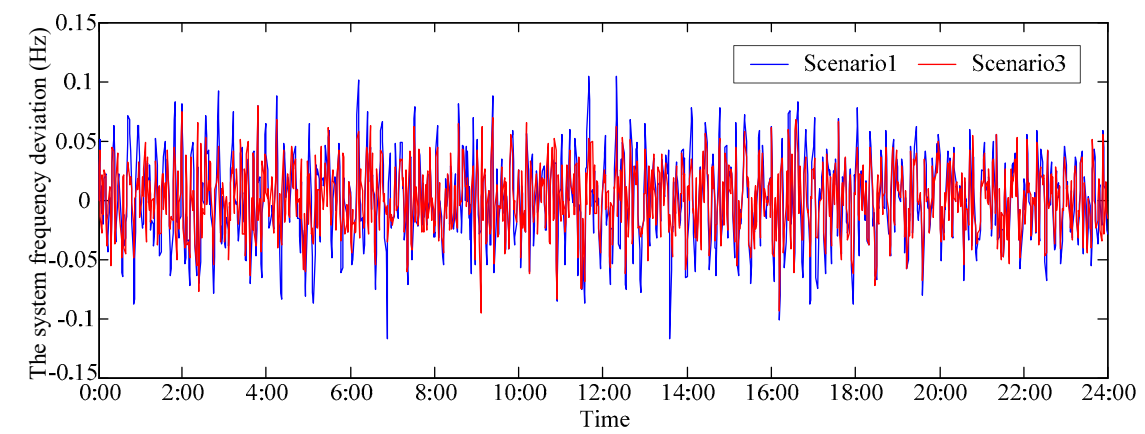

Figure 21. Comparison of the FR (frequency regulation) Effect in Scenario 1 and Scenario 3.

The proposed coordinated FR strategy is based on the real-time allocation of system FR task, which considers the FR capacity of DFIG and EV cluster to make the allocation more reasonable. In order to verify the superiority of this method, Scenario 4 is set up. In Scenario 4, the system FR task is allocated to the DFIG and EV cluster at a fixed ratio of 5:5. The FR task accomplishment situation in the two scenarios is shown in Figure 22. If the joint FR output of DFIG and EV cannot fulfill the system FR task, the shortage will be allocated to the conventional unit, and the FR task of the conventional unit is shown in Figure 22a. It can be seen that in Scenario 4, the conventional unit's FR task is obviously more than Scenario 3. This is because in the FR strategy, with fixed allocation ratio, when the FR capacity is sufficient, EV cluster and DFIG could have provided larger FR output, but the FR task is restrained by fixed allocation ratio. Due to the high climbing rate of the conventional unit, the FR task allocated to the conventional unit cannot be well accomplished, so there exists the shortage of the system FR demand power, as shown in Figure 22b. The RMS value of FR demand power shortage in Scenario 3 and Scenario 4 is 0.0325 and 0.0342 p.u., respectively. Therefore, the shortage of the system FR demand power in Scenario 4 is larger, resulting in worse FR effect.

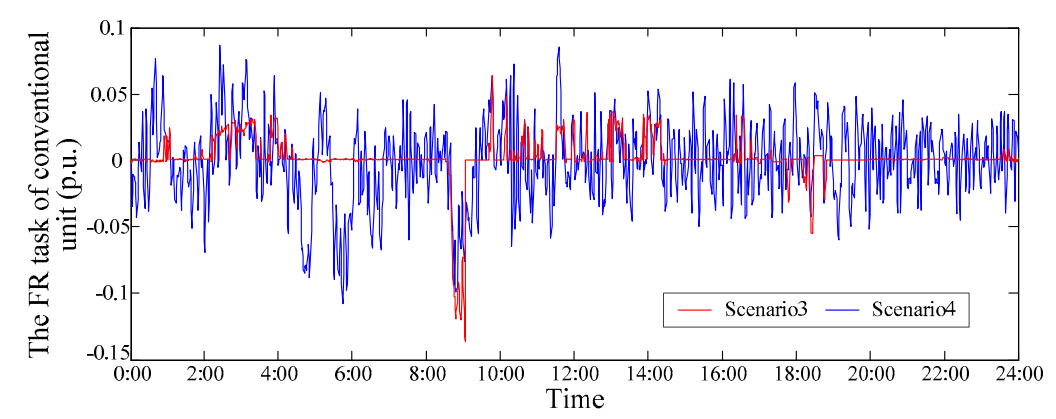

(a)

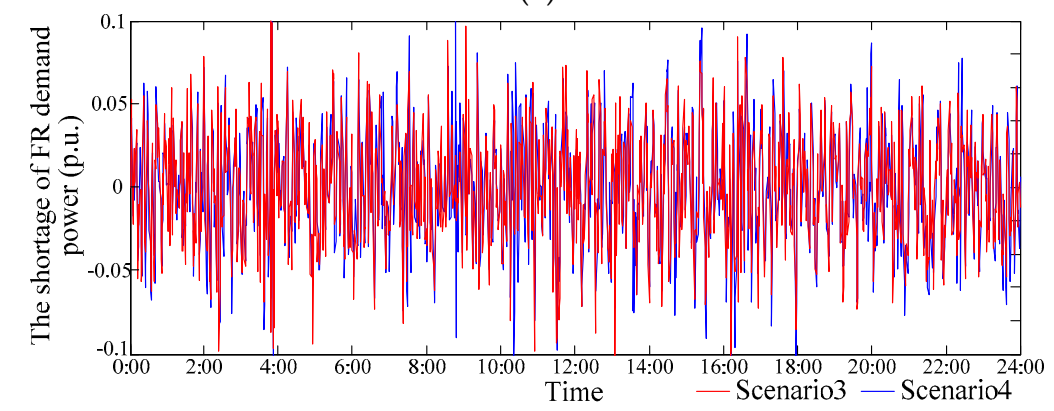

(b)

Figure 22. FR (frequency regulation) task accomplishment situation in Scenario 3 and Scenario 4. (a) The FR task of a conventional unit; (b) The shortage of system FR demand power. 
The comparison of the FR effect in Scenario 3 and Scenario 4 is shown in Figure 23. The RMS value of system frequency deviation in Scenario 3 and Scenario 4 is $0.2671 \mathrm{~Hz}$ and $0.3595 \mathrm{~Hz}$, respectively. It can be seen that the FR effect of proposed real-time allocation strategy is better than the situation of fixed allocation ratio. Therefore, the proposed coordinated FR strategy with the real-time allocation of system FR task is more reasonable and effective than the FR strategy with fixed allocation ratio.

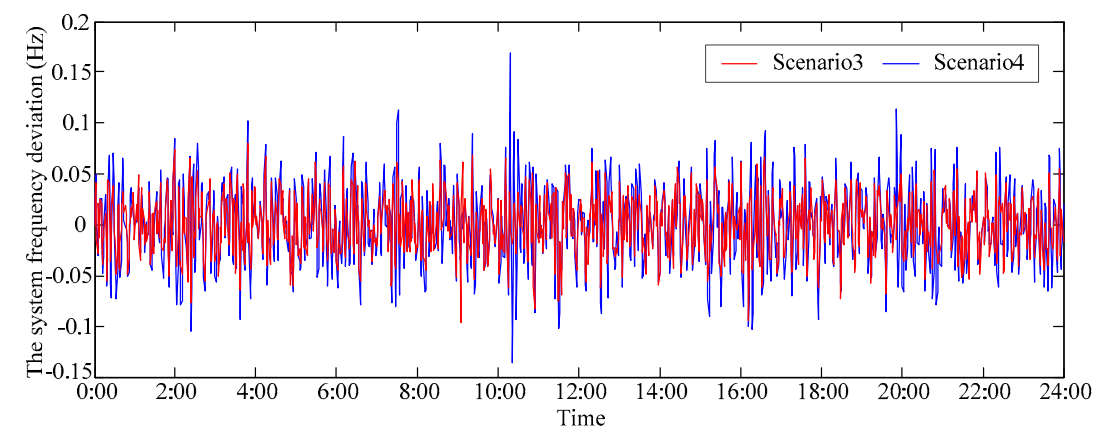

Figure 23. Comparison of the FR (frequency regulation) effect in Scenario 3 and Scenario 4.

\section{Conclusions}

With the increase in wind power penetration in the power system, WTs are required to have the ability to control active power output. In order to compensate for DFIG's weakness in FR caused by the indeterminacy and volatility of wind power, an electric vehicle is adopted as an auxiliary storage device to assist WTs to participate in system FR. This study establishes the FR model of DFIG under continuously varying WS in whole WS range, then proposes a coordinated control strategy of DFIG and EV cluster for system secondary FR with the real-time allocation of system FR task. The effectiveness of the proposed FR strategy is verified through various simulation cases based on a two-area interconnected system. Through the analysis of simulation results, the following conclusion can be obtained:

1. Considering the smooth transition of different control methods, the de-loading strategy enables DFIG to have both up-regulation and down-regulation capacity in every FR region, and the FR capacity increases with WS in low and medium WS regions while remains at the steady value in high WS region.

2. Through the FR control model based on the combination of rotor speed and pitch angle control, DFIG can effectively suppress system frequency deviation under continuously varying WS in the FR region.

3. Through the coordinated FR strategy of DFIG and EV, EV cluster can assist DFIG to participate in system secondary FR effective in the premise of meeting driver's demand, and the FR effect is better than DFIG coordinating with the conventional unit.

4. The real-time allocation strategy of system FR task, considering the FR capacity of DFIG and EV cluster, is reasonable and ensures good FR effect, which is better than the situation of fixed allocation ratio.

Author Contributions: Conceptualization, Q.Z.; Methodology, Q.Z., Y.L., and C.L.; software, C.L.; Validation, Y.L. and C.L.; Formal analysis, Y.L.; Investigation, Y.L.; Resources, D.Z.; Data curation, W.X.; Writing-Original Draft preparation, Z.D.; Writing-REVIEW and Editing, W.X.

Funding: This work was supported by the National Natural Science Foundation of China (51507022).

Conflicts of Interest: The authors declare no conflict of interest.

\section{References}

1. Bevrani, H.; Ghosh, A.; Ledwich, G. Renewable energy sources and frequency regulation: Survey and new perspectives. IET Renew. Power Gen. 2010, 4, 438-457. [CrossRef] 
2. Díaz-González, F.; Hau, M.; Sumper, A.; Gomis-Bellmunta, O. Participation of wind power plants in system frequency control: Review of grid code requirements and control methods. Renew. Sustain. Energy Rev. 2014, 34, 551-564. [CrossRef]

3. Pahasa, J.; Ngamroo, I. Coordinated control of wind turbine blade pitch angle and PHEVs using MPCs for load frequency control of microgrid. IEEE Syst. J. 2016, 10, 97-105. [CrossRef]

4. Li, Y.; Xu, Z.; Zhang, J.; Wong, K.P. Variable gain control scheme of DFIG-based wind farm for over-frequency support. Renew. Energy 2018, 120, 379-391. [CrossRef]

5. Zhang, Z.S.; Sun, Y.Z.; Lin, J.; Li, G.J. Coordinated frequency regulation by doubly fed induction generator-based wind power plants. IET Renew. Power Gen. 2012, 6, 38-47. [CrossRef]

6. Li, P.; Hu, W.; Hu, R.; Huang, Q.; Yao, J.; Chen, Z. Strategy for wind power plant contribution to frequency control under variable wind speed. Renew. Energy 2019, 139, 1226-1236. [CrossRef]

7. Zhang, W.; Fang, K. Controlling active power of wind farms to participate in load frequency control of power systems. IET Gener. Transm. Distrib. 2017, 11, 2194-2203. [CrossRef]

8. Jiang, X.; Nan, G.; Liu, H.; Guo, Z.; Zeng, Q.; Jin, Y. Optimization of Battery Energy Storage System Capacity for Wind Farm with Considering Auxiliary Services Compensation. Appl. Sci. 2018, 8, 1957. [CrossRef]

9. Tan, J.; Zhang, Y. Coordinated Control Strategy of a Battery Energy Storage System to Support a Wind Power Plant Providing Multi-Timescale Frequency Ancillary Services. IEEE Trans. Sustain. Energy 2017, 8, 1140-1153. [CrossRef]

10. He, G.; Chen, Q.; Kang, C.; Xia, Q.; Poolla, K. Cooperation of wind power and battery storage to provide frequency regulation in power markets. IEEE Trans. Power Syst. 2016, 32, 3559-3568. [CrossRef]

11. Zhang, Q.; Li, Y.; Li, C.; Li, C. Grid frequency regulation strategy considering individual driving demand of electric vehicle. Elect. Power Syst. Res. 2018, 163, 38-48. [CrossRef]

12. Vahedipour-Dahraie, M.; Rashidizaheh-Kermani, H.; Najafi, H.R.; Anvari-Moghaddam, A.; Guerrero, J.M. Coordination of EVs Participation for Load Frequency Control in Isolated Microgrids. Appl. Sci. 2017, 7, 539. [CrossRef]

13. Li, C.T.; Ahn, C.; Peng, H.; Sun, J. Synergistic control of plug-in vehicle charging and wind power scheduling. IEEE Trans. Power Syst. 2013, 28, 1113-1121. [CrossRef]

14. Arani, M.F.M.; Mohanmed, Y.A.R.I. Cooperative Control of Wind Power Generator and Electric Vehicles for Microgrid Primary Frequency Regulation. IEEE Trans. Smart Grid 2018, 9, 5677-5686. [CrossRef]

15. Varzaneh, S.G.; Abedi, M. A new simplified model for assessment of power variation of DFIG-based wind farm participating in frequency control system. Elect. Power Syst. Res. 2017, 148, 220-229. [CrossRef]

16. Fu, Y.; Zhang, X.; Hei, Y.; Wang, H. Active participation of variable speed wind turbine in inertial and primary frequency regulations. Elect. Power Syst. Res. 2017, 147, 174-184. [CrossRef]

17. Bubshait, A.; Simões, M.G. Optimal Power Reserve of a Wind Turbine System Participating in Primary Frequency Control. Appl. Sci. 2018, 8, 2022. [CrossRef]

18. Dhingra, K.; Singh, M. Frequency support in a micro-grid using virtual synchronous generator based charging station. IET Renew. Power Gen. 2018, 12, 1034-1044. [CrossRef]

19. Zecchino, A.; Prostejovsky, A.M.; Ziras, C.; Marinelli, M. Large-scale provision of frequency control via V2G: The Bornholm power system case. Elect. Power Syst. Res. 2019, 170, 25-34. [CrossRef]

20. Wikner, E.; Thiringer, T. Extending Battery Lifetime by Avoiding High SOC. Appl. Sci. 2018, 8, 1825. [CrossRef]

21. Mesbahi, T.; Khenfri, F.; Rizoug, N.; Chaaban, K.; Bartholomeüs, P.; Moigne, P.L. Dynamical modeling of Li-ion batteries for electric vehicle applications based on hybrid Particle Swarm-Nelder-Mead (PSO-NM) optimization algorithm. Elect. Power Syst. Res. 2016, 131, 195-204. [CrossRef]

22. Zhang, Q.; Liu, H.; Li, C. A hierarchical dispatch model for optimizing real-time charging and discharging strategy of electric vehicles. IEEJ Trans. Elect. Electron. Eng. 2018, 13, 537-548. [CrossRef]

23. Ota, Y.; Taniguchi, H.; Nakajima, T.; Liyanage, K.M.; Baba, J.; Yokoyama, A. Autonomous distributed V2G (vehicle-to-grid) satisfying scheduled charging. IEEE Trans. Smart Grid 2012, 3, 559-564. [CrossRef]

24. Hassan, B. Robust Power System Frequency Control; Springer: New York, NY, USA, 2009.

25. Zhang, L.; Zhao, J.; Wen, F.; Xie, Y.; Wang, J. Networked Robust Control of Electric Vehicles Based on Linear Matrix Inequalities. Autom. Elect. Power Syst. 2013, 37, 54-62, 132. (In Chinese) [CrossRef]

26. Wu, B.; Lang, Y.; Zargari, N.; Kouro, S. Power Conversion and Control of Wind Energy Systems; Wiley-IEEE Press: Hoboken, NJ, USA, 2011. 
27. Beijing Transport Institute. Beijing Transportation Development Annual Report. Available online: http: //www.bjtrc.org.cn/List/index/cid/7.html (accessed on 3 January 2019). (In Chinese).

28. Tang, D.; Wang, P. Probabilistic modeling of nodal charging demand based on spatial-temporal dynamics of moving electric vehicles. IEEE Trans. Smart Grid 2016, 7, 627-636. [CrossRef]

(C) 2019 by the authors. Licensee MDPI, Basel, Switzerland. This article is an open access article distributed under the terms and conditions of the Creative Commons Attribution (CC BY) license (http://creativecommons.org/licenses/by/4.0/). 